\title{
Bridge Integrator 2, a New Specific Target for Improving Reproductive Life Span through RPS6 and NNT
}

\section{Feng-Yu Zhu}

Nanjing Medical University

\section{Li-Li Wang}

Nanjing Medical University

\section{Tie-Gang Meng}

Guangdong Second Provincial General Hospital

\section{Zhi-Xia Yang}

Nanjing Medical University

\section{Ruo-Lei Wang}

Nanjing Medical University

\section{Gang-Yi Zhu}

Nanjing Medical University

Zhen Jin

Nanjing Medical University

\section{Lei-Lei Gao}

Nanjing Medical University

\section{Zhen-Bo Wang}

State Key Laboratory of Stem Cell and Reproductive Biology, Institute of Zoology, Chinese Academy of

Sciences https://orcid.org/0000-0001-5013-251X

\section{Qing-Yuan Sun}

Guangdong Second Provincial General Hospital

Dong Zhang ( $\square$ dong.ray.zhang@njmu.edu.cn )

Nanjing Medical University

\section{Article}

Keywords: Bin2, RPS6, NNT, primordial follicle reserve, oocyte quality

Posted Date: February 8th, 2021

DOl: https://doi.org/10.21203/rs.3.rs-144178/v1 
License: (c) (i) This work is licensed under a Creative Commons Attribution 4.0 International License. Read Full License

Version of Record: A version of this preprint was published at Nature Aging on November 11th, 2021. See the published version at https://doi.org/10.1038/s43587-021-00133-4. 


\section{Bridge Integrator 2, a New Specific Target for Improving Reproductive Life Span through RPS6 and NNT}

Feng-Yu Zhu ${ }^{1,6}$, Li-Li Wang ${ }^{1,6}$, Tie-Gang Meng ${ }^{2,3,6}$, Zhi-Xia Yang ${ }^{1,6}$, Ruo-Lei Wang ${ }^{1}$, Gang-Yi Zhu ${ }^{1}$, Zhen Jin ${ }^{1}$, Lei-Lei Gao ${ }^{1}$, Zhen-Bo Wang ${ }^{3}$, Qing-Yuan Sun ${ }^{2,5}$ and Dong Zhang $^{1,4,5}$

${ }^{1}$ State Key Lab of Reproductive Medicine, Nanjing Medical University, Nanjing, 211166, Jiangsu, China

${ }^{2}$ Fertility Preservation Lab, Reproductive Medicine Center, Guangdong Second Provincial General Hospital, Guangzhou, 510317, Guangdong, China

${ }^{3}$ State Key Lab of Stem Cell and Reproductive Biology, Institute of Zoology, Chinese Academy of Sciences, Beijing, 100101, China

${ }^{4}$ Animal Core Facility, Nanjing Medical University, Nanjing, 211166, Jiangsu, China

${ }^{5}$ To whom correspondence should be addressed to:

Dong Zhang: State Key Lab of Reproductive Medicine, Nanjing Medical University, 101 Longmiandadao, Nanjing, 211166, Jiangsu, China; dong.ray.zhang@njmu.edu.cn; Tel. +86-25-86869504; Fax.+86-25-86869504.

Qing-Yuan Sun: Fertility Preservation Lab, Reproductive Medicine Center, Guangdong Second Provincial General Hospital, 466 Xiangganzhong Road, Guangzhou, 510317, Guangdong, China; sunqy@gd2h.org.cn; Tel. +86-10-64807050; Fax. +86-10-64807050

${ }^{6}$ Equal contribution

\section{Running title}

Bin2 regulation of female fertility 


\section{Abbreviations}

Bin2: Bridge integrator 2

POF: Premature ovarian failure

PND: Post-natal-day

TF: Total follicles

PMF: PMFs

PFR: primordial follicle reserve

PF: Primary follicles

SF: Secondary follicles

AF: Antral follicles

DUF: Domain of unknown function

NNT: Nicotinamide nucleotide transhydrogenase

ROS: Reactive oxygen species

DEGs: Differentially-expressed genes

CTX: Cyclophosphamide

BPP: Bin2-penetrating peptide

Cox-1: cyclooxygenase-1

HADSCs: human adult fat stem cells

DEP: differentially-expressed protein

Herpes_ICP4_C: Herpesvirus icp4-like protein c-terminal region)

ICP4: infection cell polypeptide 4

HPO: hypothalamic-pituitary-ovary 


\section{ABSTRACT}

Female ovary is the earliest degenerated organ and it faces distinct medical disadvantages that impair primordial follicle reserve and oocyte quality. Herein, we found that bridge integrator 2 (Bin2) was predominant within mouse ovaries and oocytes, and global-knockout of Bin2 improved both female fertility and oocyte quality with healthy physiology in mice. Ovarian quantitative proteomics and phosphomics showed that Bin2 knockout specifically decreased only p-RPS6 of mTOR pathway; meanwhile, it increased nicotinamide nucleotide transhydrogenase (NNT), the free-radical detoxifier, over 6-fold. Mechanically, phosphorylation at Thr423 \& Ser424 translocated membrane Bin2 into cytoplasm to phosphorylate RPS6, while p-RPS6 bound 42-95 bp NNT UTR to inhibit NNT translation. We then synthesized a peptide (BPP) to mimic Bin2 inhibition, and found that 3-week BPP injection improved primordial follicle reserve and oocyte quality in aging or chemotherapeutics-treated mice. In all, Bin2 inhibition improve both primordial follicle reserve and oocyte quality without discernible side effects.

\section{Key words:}

Bin2, RPS6, NNT, primordial follicle reserve, oocyte quality 


\section{INTRODUCTION}

Reproductive health is important for the overall health not only of the individual self, but also of the next generation. Due to distinct physiology, women's ovaries, the core reproductive organ containing oocyte-producing primordial follicles (PMFs) and sex hormone-producing somatic cells, start to degenerate at least 20 years earlier than men's testes. Moreover, the ovary is more vulnerable than the testis to a variety of intrinsic and extrinsic adverse factors (1,2). Additionally, chemotherapy against gynecological tumors, such as ovarian cancers $(\mathrm{OC})$, always severely reduce ovarian primordial follicle reserve (PFR) and lower oocyte quality and thus cause premature ovarian failure (POF) $(3,4)$. An ideal medication would be capable of improving ovarian PFR as well as oocyte quality, and meanwhile, not interfering with normal physiology; however, such a medication has yet to be realized.

For maintaining ovarian PFR, researchers have found that the complicated "dialogue" between oocytes, granulosa cells, theca cells, and other cells is crucial for ovarian function. Many factors (ligands) and receptors play important roles in the "dialogue" process (5-8); and one of the critical downstream signal mediators of these upstream receptors is MTOR, especially mTORC1. mTORC1 targets plenty of downstream kinases to promote a variety of important processes such as protein synthesis, metabolism, ribosomal synthesis, and transcription, thus promoting follicle survival and activation (9-12). Based on these, for translational medicine, inhibition of the activating factors of the MTOR pathway can theoretically inhibit the activation of PMFs, thereby prolonging follicle reserve during the natural aging process. For example, inhibition of cyclooxygenase-1 (cox-1) can prolong the life of postnatal follicles (13). Cox-1 up-regulation is IL4-dependent and regulated by Fes-Akt-mTOR (13). Sirt1 activator SRT1720 could also improve follicle reserve and prolong ovarian life by inhibiting the MTOR pathway (14). As a direct inhibitor of MTOR, rapamycin has been shown to preserve the primordial follicle pool and thereby increases ovarian lifespan in young and aging female mice (15). Caloric restriction can also protect follicle reserve by inhibiting the MTOR pathway (16). However, despite the positive 
roles of selective inhibition of mTOR pathway activators, several concerns still remain. Firstly, kinases within mTOR pathway are mostly universally active and important in all tissues, inhibition of these kinases could disrupt normal biological processes and substantially hurt overall body health. For example, rapamycin is also an immunosuppressant and might lead to adverse metabolic reactions (17). Secondly, patients usually have medium to high levels of drug-related adverse reactions (such as diarrhea and nausea) to these chemicals (18). Thirdly, inhibition of these mTOR activators are "double-edged swords," in that an excessive inhibition could reduce follicle survival and lower oocyte quality since they are critical for these aspects (5-12). Besides, other PFR-improving strategies beyond inhibition of mTOR activators, such as activating the SIRT1/FOXO1 signaling through hADSC-secreted HGF and bFGF (19) or up-regulating erk phosphorylation through Ptk2b knockout (20), could not specifically target ovaries as well and carry the risk of tumorigenesis $(21,22)$.

For improving oocyte quality, although the physiological level of ROS (reactive oxygen species) is essential for follicular rupture and oocyte maturation, excessive ROS generation under disadvantageous circumstance such as psychological stress, aging, or chemotherapy is detrimental (23). Elevated ROS could conduce to enzyme inactivation, lipid peroxidation, ATP depletion, mitochondrial disruption, and meiotic spindle defects, finally result in decreased oocyte quality, apoptosis and reduced fertility (24). Therefore, many translational researches supported that oocyte quality could be improved by reducing ROS. For example, Nicotinamide Mononucleotide (NMN) supplementation eliminated accumulated ROS to suppress apoptosis in aged oocytes $(25,26)$. CoQ10 supplementation decreased ROS levels and prevented abnormal mitochondrial distribution and in oocytes of obese mice (27). BAT transplantation could also improve oocyte quality of aging mice (28). However, the upper and most of other anti-oxidants, and BAT transplantation could not increase PFR, and non-enzymatic anti-oxidants are less efficient than enzymatic ROS detoxifiers such as SOD (superoxide dismutase) and Gpx (glutathione peroxidase) $(23,25,26)$. It has been reported that supplement of melatonin (29), quercetin (30), or resveratrol (31) could improve both PFR and oocyte quality by reducing ROS and 
apoptosis. However, melatonin can inhibit the hypothalamic-pituitary-gonadal axis or directly act on the ovary to reduce the content of androgens, estrogens and progesterone (32). Quercetin is reported to be mutagenic in some studies (33). Resveratrol has to be daily-taken for as long as 12 months in mice (about 40 years in human) to show significant increase in PFR (31). In addition, the clinical efficacy of all these treatments remains controversial.

In the current study, we found that a novel target, Bin2, might be a critical regulator of both PFR and oocyte quality. Since Bin2 is fairly dominant in ovaries and oocytes, target inhibition of Bin2 to prolong female fertility and improve oocyte quality did not affect normal physiology.

\section{RESULTS}

\section{Bin2 is rich in ovaries \& oocytes and essential for primordial follicle activation}

Many important cancer-related genes are essential for ovarian function and female fertility. We herein have called these cancer/ovary genes, in concert with reported cancer/testis genes. Bin2 (Bridge integrator 2, gene ID 668218) was also named BRAP1 (breast cancer-associated protein 1). It has been reported that in diverse human cancers, including breast cancer and ovarian cancer, Bin2 has over 40 enhanced post-translational modification sites, among which most are phosphorylation sites (https://www.phosphosite.org/proteinAction?id=9451\&showAllSites=true), suggesting that Bin2 might be over-activated through hyper-phosphorylation to promote tumorigenesis. We primarily showed that Bin2 overexpression in human epithelial ovarian cancer (EOC) cells significantly up-regulated p-RPS6 of the mTOR pathway while not altering p-Akt (Fig. 1A), while Bin2 knockdown in human epithelial ovarian cancer (EOC) cells significantly down-regulated p-RPS6 while also not altering p-Akt (Fig. 1B). p-RPS6 over-activation is a major cause of premature activation of the primordial follicle pool (34). Bin2's function in female fertility is as yet unknown.

We first found that Bin2 protein was the richest in ovaries, as compared with other 
major tissues (Fig. 1C), and Bin2 protein level sharply increased as initial follicle recruitment occurred (Fig. 1D). Bin2 mRNA level in oocytes was much higher than the other two Bin family members, Bin1 and Bin3 (Fig. 1E) and remained constant during meiosis (Fig. 1F). Moreover, Bin2 protein was more dominant in oocytes than in granulosa cells (Fig. $1 \mathrm{G}$ and $1 \mathrm{H}$ ). These indicated that Bin2 might have important functions within ovaries, and primarily within oocytes.

To uncover its function, we used the cas9 technique to delete the whole exon 9 (83 bases) of Bin2 genome to obtain Bin2 global knockout mice (Fig 1I). Western blot showed that Bin2 protein completely disappeared in the knockout ovaries (Fig. 1J). Surprisingly, Bin2 knockout did not affect ovary weight, body weight, and ovary/body weight ratio; moreover, Bin2 knockout did not affect oocyte maturation or zygote development (Suppl. Fig. 1A-E). While the 60-week-long fertility assay showed that both the cumulative litter number per mouse and the average litter size significantly increased (Fig 1K-N). Further continuous follicle count showed that from 6 months old, both the total follicle number and the primordial follicle number significantly were significantly higher in Bin2-knockout ovaries (Fig. 1O-R). Additionally, Bin2 knockout did not affect major immunity or inflammation indexes including C-reactive protein (CRP), CD4, and IL6 within plasma (Suppl Fig. 2A and 2B) and ovaries (Suppl. Fig. $2 \mathrm{C}$ and 2D) when compared with the control. These results indicated that Bin2 depletion could significantly improve primordial follicle survival and increase fertility without affecting the overall health of the female mice.

\section{Phosphorylation at Thr423 \& Ser424 translocated Bin2 into cytoplasm to activate RPS6}

From the above results, Bin2 might be a novel target for the safe augmentation of primordial follicle reserve and ovarian lifespan. Since Bin2 is highly conserved between diverse mammalian species, including mouse and human, investigating the mechanism by which Bin2 functions in female mice might ultimately contribute to the improvement of ovarian performance in human females. We used TMT-labeled quantitative phosphomics to systematically identify differentially phosphorylated 
proteins $(\geq 1.2$-fold or $\leq 0.833$-fold) between control and Bin2-knockout 3-week-old ovaries and carefully observed pathways known to be involved in primordial follicle survival and activation and oocyte quality maintenance-for example, mTOR pathway (Fig. 2A). We found that only RPS6 of the mTOR pathway had significantly decreased phosphorylation (Fig. 2B and 2C), which was verified by Western blot and immunofluorescence (Fig. 2D-F). In addition, TMT-labeled quantitative proteomics (Fig. 2G) and RNA sequencing (Fig. 2H) showed that major components of the mTOR pathway did not change at transcriptional or translational levels. This suggests that Bin2 might interact with and regulate RPS6 at a post-translational (phosphorylation) level.

However, Bin2 was enriched on the membrane, while RPS6 was within cytoplasm, rendering it unreasonable to conclude that Bin2 interacts with RPS6. Notably, Bin2 has many predicted phosphorylation sites, so we hypothesized that Bin2 might be activated through phosphorylation and translocated into cytoplasm. We identified several phosphorylation sites (Suppl. Table 1); among these, Thr423 \& Ser424 were in the c-terminal domain of unknown function (DUF domain). We also found that these two residues and the adjacent residues are highly conserved between humans, rats, mice, rhesus monkeys, and Cynomolgus macaques (Fig. 3A and 3B), so we hypothesized that these two residues might be the key residues for the activation and translocation of Bin2. We generated a phosphorylation antibody using a core phospho peptide "QSKRAASIQR(pThr) (pSer)A" and verified its specificity through Bin2 siRNA and corresponding non-phospho peptide "QSKRAASIQRTSA" (Fig 3C and 3D). Immunostaining showed that Bin2 was enriched on the oocyte membrane, while $p$-Bin2 was enriched within the nucleus at the GV stage and within spindles during meiosis, which was quite similar to p-RPS6 (Fig 3E and 3F, Suppl. Fig. 3).

Next, to further verify the relationship between Bin2 and RPS6, we injected the non-phospho peptide "QSKRAASIQRTSA" into the GV oocytes and speculated that this peptide could compete with the endogenous Bin2, thereby decreasing the phosphorylation of endogenous Bin2. We found that $p$-Bin2 level significantly decreased, while p-RPS6 level also significantly decreased (Fig 3G). In all, these 
results supported the conclusion that $\mathrm{p}$-Bin2 could specifically activate (phosphorylate) RPS6 without affecting other components of the mTOR pathway.

\section{In vitro characterization of Bin2 phosphorylation activity on rps6}

We attempted to directly verify the relationship between Bin2 and RPS6 and the key roles of Thr423 and Ser424 on Bin2 activation. We used the Bac-to-bac system to express full-length wild-type Bin2 (Bin2-WT) and a Thr423 and Ser424 to Ala423 and Ala 424 mutant Bin2 (Bin2-AA) (Fig. 4A). We also expressed and purified RPS6 from E. coli. We found that the primary advantage of bacteria-derived RPS6 was that it was fully inactive (non-phosphorylated). Firstly, Bin2-WT can phosphorylate RPS6 more effectively than an equal amount of Bin2-AA (Fig. 4B). Next, in vitro dose-dependent phosphorylation assay showed that Bin2-WT could phosphorylate RPS6 much more strongly than Bin2-AA (Fig. 4C and 4D). Immunostaining on bacularvirus-infected sf9 cells showed that Bin2-WT localized both on the membrane and within the cytoplasm, while Bin2-AA almost exclusively localized on the membrane; accordingly, p-RPS6 intensity in Bin2-WT bacularvirus-infected sf9 cells was significantly higher than in Bin2-AA bacularvirus-infected sf9 cells (Fig. 4E and $4 \mathrm{~F})$.

Next, we used the Bac-to-bac system to express and purify two different motifs of Bin2, BAR (1-240 AA) and DUF (domain of unknown function, 241-505 AA), and examined the difference between them (Fig. 4G). Firstly, we found that Bin2-DUF could phosphorylate RPS6 more effectively than an equal amount of Bin2-BAR (Fig. 4H). Secondly, in vitro dose-dependent phosphorylation assay showed that Bin2-DUF could phosphorylate RPS6 much more strongly than Bin2-BAR (Fig. $4 \mathrm{I}$ and $4 \mathrm{~J}$ ). These results further supported the conclusion that Bin2 phosphorylation at Thr423 and Ser424 is the primary mechanism for activating RPS6.

\section{Bin2 knockout promotes nicotinamide nucleotide} transhydrogenase translation through down-regulating p-RPS6

TMT-labelled quantitative proteomics (proteomics were used to normalize the phosphomics) between control and Bin2-KO 3-week-old ovaries showed that, among 
the differentially expressed gross proteins $¥ 1.2$-fold or $\leq 0.833$-fold), nicotinamide nucleotide transhydrogenase (NNT) was most up-regulated (>6-fold, Fig. 5A and 5B, arrow pointed). We have reliably verified the NNT increment through blot (Fig. 5C and 5D). In the meantime, NNT mRNA remained unchanged (Fig. 5E and 5F). Moreover, RPS6 knockdown also up-regulated NNT protein level while keeping NNT mRNA level (Fig. 5G and 5H). NNT regenerated NADP into NADPH and could act as free-radical detoxifier (35), and it was shown that elevated NNT improved oocyte quality and developmental competence (36). Thus, we hypothesized that Bin2 knockout could improve oocyte quality through NNT. Live dye staining showed that the ROS level significantly decreased (Fig. $5 \mathrm{I}$ and $5 \mathrm{~J}$ ), while the JC-1 level significantly increased (Fig. $5 \mathrm{~K}$ and $5 \mathrm{~L}$ ) within Bin2-knockout oocytes. These indicated that Bin2 knockout could even improve oocyte quality.

Next, we tried to address whether p-Bin2 or p-RPS6 binds the translation-regulating regions of NNT to inhibit its translation. We found that NNT 5' UTR (untranslated region) are very conserved between multiple species including humans, rats, mice, rhesus monkeys, and Cynomolgus macaques (Supple. Fig. 4). We subdivided NNT 5' UTR into seven shorter or four longer regions (Fig. 5M). We used Bin2 and RPS6 purified from sf9 cells, since these proteins were verified to have phosphorylated portions (Figure 5N and 5O). Protein-RNA UV crosslinking and gel shift assay showed that p-RPS6 preferred to bind short regions 3 and 4, from 42 to 95 bp of NNT 5' UTR, instead of other UTR or CDS regions (Fig. 5P and 5Q, arrow pointed), whereas p-Bin2 did not bind any shorter or longer regions (Fig. 5R and $5 S$ ). These results suggested that $p$-Bin2 negatively regulates NNT translation through p-RPS6, while p-RPS6 negatively regulates NNT translation by directly binding NNT 5' UTR region.

\section{Inhibition of Bin2 phosphorylation in vivo prolongs ovarian lifespan in aging mice}

As stated above, female ovarian function and fertility generally begins to drop at 35 years of age. The existing strategies for prolonging ovarian lifespans in mice have 
demonstrated several side effects. Since Bin2 knockout increased fertility without discernible side effects, we proposed that Bin2 might be a novel target for safely prolonging ovarian function and female fertility. We planned to use 8-month-old aging mice as models to mimic 35-year-old women (Fig. 6A), and we tested whether inhibiting Bin2 phosphorylation through the non-phospho peptide "QSKRAASIQRTSA" could largely mimic the effects of Bin2 knockout in mice.

To facilitate the permeation of this peptide in vivo, we fused PEP1, the known cell-penetrating peptide (37), to this peptide and renamed it as BPP (Bin2-penetrating peptide, Fig. 6B). We firstly verified that FITC-labeled BPP can efficiently enter ovaries (Fig. 6C) or in vitro-cultured oocytes and A2780 cells (Suppl. Fig. 5A and 5B), and that both p-Bin2 and p-RPS6 significantly decreased within ovaries (Fig. 6D). We also showed that BPP had typical dose-dependent and time-dependent dynamics within the plasma (Suppl. Fig. 6A and 6B). Next, after a 3-week-long BPP injection cycle (once a day) on 8-month-old aging mice, fertility assay from 36 weeks old to 63 weeks old showed that the number of cumulative pups significantly increased (Fig. 6E-G). Continuous follicle counting showed that total follicles and PMFs, and primary follicles (Fig 6H and 6I, Supple. Fig. 7A and 7B) all increased. Accordingly, p-RPS6 also significantly decreased (Fig. 6J and 6K), while NNT level within the oocytes significantly increased (Fig. 6L and 6M). In addition, major organ weight (Suppl. Fig. $8 \mathrm{~A}$ and $8 \mathrm{~B}$ ) and major blood biochemical indexes (Suppl. Fig. 9) assays did not change after Bin2 inhibition. These results indicated that ovary function in aging mice have been improved by Bin2 inhibition without discernible side effects.

Finally, we examined how well BPP could revive ovarian function in the aging mice at the transcriptional level. RNA sequencing showed that at the fold threshold of $\mid \log 2$ (2-month/9-month)| $\geq 2$, there were 1858 DEGs (differentially-expressed genes) between 2-month ovaries and 9-month ovaries; at the fold threshold of $\mid \log 2$ (9-month-BPP/9-month) $\mid \geq 2$, there were 1138 DEGs (differentially-expressed genes) between 9-month-BPP ovaries and 9-month ovaries; and between these two clusters of DEGs, 622 DEGs overlapped, suggesting that BPP injection could significantly recover the 9-month ovarian profile, bringing it close to the 2-month 
ovarian profile. (Fig 6N and 60). KEGG analysis showed that multiple follicle survival, oocyte quality, or oocyte steriodgenesis-related pathways were among the top 10 enriched signaling pathways (Fig 6P).

\section{Inhibiting Bin2 phosphorylation in vivo protects ovaries from chemotherapy-induced premature ovarian failure}

It is known that chemotherapy can cause premature ovarian failure (POF); one of the major mechanisms of this is mTOR pathway over-activation. Therefore, mTOR inhibitors could rescue ovarian function and fertility $(13,14,17)$. However, mTOR-inhibiting chemicals also have multiple side effects $(17,18)$. We wanted to test whether BPP could also rescue chemotherapy-induced POF safely and effectively. We used 2-month-old mice to mimic pubescent women (about 16-year-old, Fig. 6A).

Western blot showed that a harsh, three-week-long cyclophosphamide (CTX) treatment cycle significantly elevated p-RPS6, while BPP treatment reduced p-RPS6 level close to the control level (Fig. 7A). Moreover, CTX treatment significantly decreased oocyte NNT level, while BPP injection recovered NNT level close to the control (Fig. 7B and 7C). Consequently, fertility assay from 4 months old to 9 months old showed that CTX treatment almost induced complete infertility, while BPP significantly recovered fertility, although still less than that of the control (Fig. 7D-F). Continuous follicle counting showed that CTX treatment sharply decreased numbers of PMFs and growing follicles, while BPP significantly up-regulated follicle numbers, although still less than those of the control (Fig. 7G and 7H). These results indicated that BPP could effectively preserve ovarian primordial follicle pool and simultaneously maintain oocyte quality during chemotherapy.

\section{DISCUSSION}

Bin2 belongs to the BAR family proteins that are mostly involved in the phagocytosis of synaptic vesicles, the regulation of microfilaments, cell division, and migration in different cells $(38,39)$. And the non-conserved c-terminal region might explain the functional diversity of different BAR proteins $(38,39)$. An in-vitro study has shown that human Bin2 knockdown led to decreased leukocyte migration, pseudopod 
density, and pseudopod kinetics (40). Recently, Bin2 was shown to play crucial roles in platelet activation in thrombosis and thrombo-inflammation (41). However, there have been no reports on its function in reproduction. In NCBI, the c-terminal region of human Bin2 contains ICP4 (infection cell polypeptide 4), a phosphoprotein, suggesting that Bin2 could be activated or deactivated through phosphorylation or dephosphorylation (respectively). We have independently characterized two key phosphorylation sites (Thr423 and Ser424) within the c-terminal region (we temporarily named it DUF, Fig. 4). And notably, although these regions are highly variable among humans, cynomolgus monkeys, macaque monkeys, mice, and rats, the peptide sequences around Thr423 and Ser424 are highly conserved (Supple. Fig. 10 , shadowed in the rectangle), suggesting that the conserved activation/inactivation mechanism might rely on these two residues. On the other side, human Bin2 is also called BRAP-1 (Breast cancer-associated protein 1) and has 40 reinforced post-translational modification sites in diverse human tumors, suggesting that its over-activation may promote cell proliferation. In the present study, we found that Bin2 knockout specifically down-regulated p-RPS6 of the mTOR pathway. RPS6 hyper-phosphorylation is closely related to follicular over-activation (34), suggesting that targeted down-regulating p-RPS6 by Bin2 knockout or inhibition could inhibit primordial follicle activation, thereby increase PFR. These conclusions are supported by multiple results in our present study.

RPS6 is a component of ribosome 40S subunit. The peculiar feature which differentiates RPS6 from other ribosomal proteins is that it can bind chromatin as well as localize within matrix and cytoplasm (42). Conceivably, it can regulate various important cellular processes. Actually, RPS6 is known to be regulated by multiple kinases and phosphatases (43-45). In the present study, we showed that Bin2 might be a novel kinase that directly phosphorylates RPS6 (Fig. 4). Thus, Bin2 knockout or inhibition is predicted to moderately down-regulate p-RPS6. On the other side, RPS6 itself was also essential for primordial follicle survival; excessive RPS6 suppression or Rps6 knockout caused loss of PMFs (45), so the extent of RPS6 reduction must be moderate to ensure both the survival and increase of PMFs. In our study, Bin2 
knockout or inhibition appeared to cause an optimum p-RPS6 reduction, which is the critical base of the duet function of Bin2.

The main function of NNT (nicotinamide nucleotide transhydrogenase) is to regenerate NADP into NADPH and maintain the mitochondrial redox balance (35); NNT deficiency causes vascular ROS production and exacerbates atherosclerotic plaque development (46). On the other side, NNT levels decrease as aging and mitochondrial NAD levels also decrease in somatic cells, while NNT overexpression could restore NAD levels and promote the reprogramming of aged somatic cells (47). In our study, Bin2 knockout and inhibition both greatly increased NNT, which might be

able to protect oocyte quality even in cases in which p-RPS6 is harshly reduced. Moreover, we discovered that $\mathrm{p}$-Bin2 regulates NNT translation through $\mathrm{p}$-RPS6, and thus it is likely p-RPS6 that directly binds the $42-95$ bp NNT 5' UTR region to inhibit NNT translation. Therefore, we provided a clear model for how Bin2 knockdown or inhibition can improve oocyte quality through p-RPS6 and NNT.

In all, we showed that Bin2 knockout or inhibition could have a duet effect (Fig. 8). At the post-translational level, p-Bin2 specifically activated p-RPS6 of mTOR; thus,

p-Bin2 inhibition improved the PFR by specifically and moderately down-regulating p-RPS6. At the translational level, p-RPS6 binds to $42-95$ bp of NNT 5' UTR mRNA to inhibit NNT translation; thus, $p$-Bin2 inhibition up-regulated NNT protein levels to improve oocyte quality. Since Bin2 is fairly predominant in ovaries \& oocytes, the effect of $p$-Bin2 inhibition might be limited primarily in ovaries \& oocytes. Therefore, compared with the current strategies, Bin2 could be a novel, specific, and safe target for improving PFR and oocyte quality (Fig. 8, Supple. Fig. 11).

\section{ACKNOWLEDGEMENT}

This work is financially supported by the General Program of the National Natural Science Foundation of China (Grant No: 32070840) to Dong Zhang, and the National Key Research and Development Program of China (Grant No: 2017YFC1001503 and 2018YFC1003400 to Dong Zhang, and 2018YFA0107701 to Qing-Yuan Sun). 


\section{AUTHOR CONTRIBUTIONS}

Dong Zhang, Qing-Yuan Sun, and Zhi-Xia Yang designed the research. Tie-Gang Meng made the Bin2-knockout mice under the direction of Qing-Yuan Sun; Feng-Yu Zhu, Li-Li Wang, and Tie-Gang Meng performed most of the experiments. Ruo-Lei Wang assisted during the experiments. Gang-Yi Zhu, Zhen Jin, and Lei-Lei Gao conducted many preliminary works of Bin2. Feng-Yu Zhu and Li-Li Wang did all data analysis and prepared figures under the direction of Dong Zhang, Qing-Yuan Sun, and Zhi-Xia Yang. Dong Zhang wrote the manuscript, Qing-Yuan Sun and Zhi-Xia Yang proofread and gave advice. Professor Zhen-Bo Wang gave many valuable advices and supports during the research.

\section{AVAILABILITY OF DATA AND MATERIAL}

The data that support the findings of this study are available from the corresponding author upon reasonable request. Supplementary datasets 1-5 have been deposited into Zendo (https://zenodo.org/deposit/4006605), the DOI is 10.5281/zenodo.4006605. Accession codes for all other relevant data will be available before publication.

\section{REFERENCE}

1. Laisk $T$, Tšuiko $O$, Jatsenko $T$, Hõrak $P$, Otala $M$, Lahdenperä $M$, Lummaa $V$, Tuuri T, Salumets A, Tapanainen JS. Demographic and evolutionary trends in ovarian function and aging. Hum Reprod Update. 2019 25(1):34-50.

2. McCracken $\mathrm{K}$, Nahata L. Fertility preservation in children and adolescents: current options and considerations. Curr Opin Obstet Gynecol. 2017 29(5):283-288.

3. Lawrenz B, Henes M, Neunhoeffer E, Fehm T, Lang P, Schwarze CP. Fertility preservation in girls and adolescents before chemotherapy and radiation - review of the literature. Klin Padiatr. 2011 223(3):126-130.

4. Spears N, Lopes F, Stefansdottir A, Rossi V, De Felici M, Anderson RA, Klinger FG. Ovarian damage from chemotherapy and current approaches to its protection. Hum Reprod Update. 2019 25(6):673-693. 
5. Chang HM, Wu HC, Sun ZG, Lian F, Leung PCK. Neurotrophins and glial cell line-derived neurotrophic factor in the ovary: physiological and pathophysiological implications. Hum Reprod Update. 2019 25(2):224-242.

6. Liu Y, Du SY, Ding M, Dou X, Zhang FF, Wu ZY, Qian SW, Zhang W, Tang QQ, Xu CJ. The BMP4-Smad signaling pathway regulates hyperandrogenism development in a female mouse model. J Biol Chem. 2017 292(28):11740-11750.

7. Saatcioglu HD, Cuevas I, Castrillon DH. Control of Oocyte Reawakening by Kit. PLoS Genet. 2016 12(8):e1006215.

8. Baumgarten SC, Armouti M, Ko C, Stocco C. IGF1R Expression in Ovarian Granulosa Cells Is Essential for Steroidogenesis, Follicle Survival, and Fertility in Female Mice. Endocrinology. 2017 158(7):2309-2318.

9. Chen J, Torcia S, Xie F, Lin CJ, Cakmak H, Franciosi F, Horner K, Onodera C, Song JS, Cedars MI, Ramalho-Santos M, Conti M. Somatic cells regulate maternal mRNA translation and developmental competence of mouse oocytes. Nat Cell Biol. 2013 15(12):1415-1423.

10. De Felici M, Lobascio AM, Klinger FG. Cell death in fetal oocytes: many players for multiple pathways. Autophagy. 2008 4(2):240-242.

11. Guo J, Zhang T, Guo Y, Sun T, Li H, Zhang X, Yin H, Cao G, Yin Y, Wang H, Shi L, Guo X, Sha J, Eppig JJ, Su YQ. Oocyte stage-specific effects of MTOR determine granulosa cell fate and oocyte quality in mice. Proc Natl Acad Sci U S A. 2018 115(23):E5326-E5333.

12. Guo J, Shi L, Gong X, Jiang M, Yin Y, Zhang X, Yin H, Li H, Emori C, Sugiura K, Eppig JJ, Su YQ. Oocyte-dependent activation of MTOR in cumulus cells controls the development and survival of cumulus-oocyte complexes. J Cell Sci. 2016 129(16):3091-3103.

13. Yang WL, Cai KQ, Smedberg JL, Smith ER, Klein-Szanto A, Hamilton TC, Xu XX. A reduction of cyclooxygenase 2 gene dosage counters the ovarian morphological aging and tumor phenotype in Wv mice. Am J Pathol. 2007 170(4):1325-1336. 
14. Zhou XL, Xu JJ, Ni YH, Chen XC, Zhang HX, Zhang XM, Liu WJ, Luo LL, Fu YC. SIRT1 activator (SRT1720) improves the follicle reserve and prolongs the ovarian lifespan of diet-induced obesity in female mice via activating SIRT1 and suppressing mTOR signaling. J Ovarian Res. 2014 7:97.

15. Dou X, Sun Y, Li J, Zhang J, Hao D, Liu W, Wu R, Kong F, Peng X, Li J. Short-term rapamycin treatment increases ovarian lifespan in young and middle-aged female mice. Aging Cell. 2017 16(4):825-836.

16. Li L, Fu YC, Xu JJ, Lin XH, Chen XC, Zhang XM, Luo LL. Caloric restriction promotes the reserve of follicle pool in adult female rats by inhibiting the activation of mammalian target of rapamycin signaling. Reprod Sci. 2015 22(1):60-67.

17. Goldman, K. N., Chenette, D., Arju, R., Duncan, F. E., Keefe, D. L., Grifo, J. A., \& Schneider, R. A.-O. mTORC1/2 inhibition preserves ovarian function and fertility during genotoxic chemotherapy. Proc Natl Acad Sci U S A 2017 114(12):3186-3191.

18. Blagden S, Omlin A, Josephs D, Stavraka C, Zivi A, Pinato DJ, Anthoney A, Decordova S, Swales K, Riisnaes R, Pope L, Noguchi K, Shiokawa R, Inatani M, Prince J, Jones K, Twelves C, Spicer J, Banerji U. First-in-human study of CH5132799, an oral class I PI3K inhibitor, studying toxicity, pharmacokinetics, and pharmacodynamics, in patients with metastatic cancer. Clin Cancer Res. 2014 20(23):5908-5917.

19. Ding C, Zou Q, Wang F, Wu H, Wang W, Li H, Huang B. HGF and BFGF Secretion by Human Adipose-Derived Stem Cells Improves Ovarian Function During Natural Aging via Activation of the SIRT1/FOXO1 Signaling Pathway. Cell Physiol Biochem. 2018 45(4):1316-1332.

20. Cong Y, Wu H, Bian X, Xie Q, Lyu Q, Cui J, Suo L, Kuang Y. Ptk2b deletion improves mice folliculogenesis and fecundity via inhibiting follicle loss mediated by Erk pathway. J Cell Physiol. 2020 Jul 1. doi: 10.1002/jcp.29914. Online ahead of print. 
21. Wang Y, Liu J, Jiang Q, Deng J, Xu F, Chen X, Cheng F, Zhang Y, Yao Y, Xia Z, Xu X, Su X, Huang M, Dai L, Yang Y, Zhang S, Yu D, Zhao RC, Wei Y, Deng H. Human Adipose-Derived Mesenchymal Stem Cell-Secreted CXCL1 and CXCL8 Facilitate Breast Tumor Growth By Promoting Angiogenesis. Stem Cells. 2017 35(9):2060-2070.

22. Rodriguez-Aguayo C, Bayraktar E, Ivan C, Aslan B, Mai J, He G, Mangala LS, Jiang D, Nagaraja AS, Ozpolat B, Chavez-Reyes A, Ferrari M, Mitra R, Siddik ZH, Shen H, Yang X, Sood AK, Lopez-Berestein G. PTGER3 induces ovary tumorigenesis and confers resistance to cisplatin therapy through up-regulation Ras-MAPK/Erk-ETS1-ELK1/CFTR1 axis. EBioMedicine. 2019 40:290-304.

23. Kala M, Shaikh MV, Nivsarkar M.

Equilibrium between anti-oxidants and reactive oxygen species: a requisite for oocyte development and maturation. Reprod Med Biol. 2016 16(1):28-35.

24. Singh AK, Chattopadhyay R, Chakravarty B, Chaudhury K. Markers of oxidative stress in follicular fluid of women with endometriosis and tubal infertility undergoing IVF. Reprod Toxicol. 2013 42:116-124.

25. Miao Y, Cui Z, Gao Q, Rui R, Xiong B. Nicotinamide Mononucleotide Supplementation Reverses the Declining Quality of Maternally Aged Oocytes. Cell Rep. 2020 32(5):107987.

26. Bertoldo MJ, Listijono DR, Ho WJ, Riepsamen AH, Goss DM, Richani D, Jin XL, Mahbub S, Campbell JM, Habibalahi A, Loh WN, Youngson NA, Maniam J, Wong ASA, Selesniemi K, Bustamante S, Li C, Zhao Y, Marinova MB, Kim LJ, Lau L, Wu RM, Mikolaizak AS, Araki T, Le Couteur DG, Turner N, Morris MJ, Walters KA, Goldys E, O'Neill C, Gilchrist RB, Sinclair DA, Homer HA, Wu LE. NAD+ Repletion Rescues Female Fertility during Reproductive Aging. Cell Rep. 2020 30(6):1670-1681.

27. Boots CE, Boudoures A, Zhang W, Drury A, Moley KH.

Obesity-induced oocyte mitochondrial defects are partially prevented and 
rescued by supplementation with co-enzyme Q10 in a mouse model. Hum Reprod. 2016 31(9):2090-2097.

28. Chen LJ, Yang ZX, Wang Y, Du L, Li YR, Zhang NN, Gao WY, Peng RR, Zhu FY, Wang LL, Li CR, Li JM, Wang FQ, Sun QY, Zhang D. (2019) Single xenotransplant of rat brown adipose tissue prolonged the ovarian lifespan of aging mice by improving follicle survival. Aging Cell. 2019 18(6):e13024.

29. Jang H, Lee OH, Lee Y, Yoon H, Chang EM, Park M, Lee JW, Hong K, Kim JO, Kim NK, Ko JJ, Lee DR, Yoon TK, Lee WS, Choi Y. Melatonin prevents cisplatin-induced primordial follicle loss via suppression of PTEN/AKT/FOXO3a pathway activation in the mouse ovary. J Pineal Res 2016 60(3):336-347.

30. Naseer Z, Ahmad E, Epikmen ET, Uçan U, Boyacioğlu M, Ipek E, Akosy M. Quercetin supplemented diet improves follicular development, oocyte quality, and reduces ovarian apoptosis in rabbits during summer heat stress. Theriogenology. 2017 96:136-141.

31. Liu M, Yin Y, Ye X, Zeng M, Zhao Q, Keefe DL, Liu L. Resveratrol protects against age-associated infertility in mice. Hum Reprod. 2013 28(3):707-717.

32. Shi L, Li N, Bo L, Xu Z. Melatonin and hypothalamic-pituitary-gonadal axis. Curr Med Chem. 2013 20(15):2017-2031.

33. Resende FA, Vilegas W, Dos Santos LC, Varanda EA. Mutagenicity of flavonoids assayed by bacterial reverse mutation (Ames) test. Molecules 2012 17(5):5255-5268.

34. Reddy P, Liu L, Adhikari D, Jagarlamudi K, Rajareddy S, Shen Y, Du C, Tang W, Hämäläinen T, Peng SL, Lan ZJ, Cooney AJ, Huhtaniemi I, Liu K. Oocyte-specific deletion of Pten causes premature activation of the primordial follicle pool. Science. 2008 319(5863):611-613.

35. Ho HY, Lin YT, Lin G, Wu PR, Cheng ML. Nicotinamide nucleotide transhydrogenase (NNT) deficiency dysregulates mitochondrial retrograde signaling and impedes proliferation. Redox Biol. 2017 12:916-928. 
36. Single-Cell Transcriptomics of Human Oocytes: Environment-Driven Metabolic Competition and Compensatory Mechanisms During Oocyte Maturation. Zhao H, Li T, Zhao Y, Tan T, Liu C, Liu Y, Chang L, Huang N, Li C, Fan Y, Yu Y, Li R, Qiao J. Antioxid Redox Signal. 2019 30(4):542-559.

37. Morris MC, Depollier J, Mery J, Heitz F, Divita G. A peptide carrier for the delivery of biologically active proteins into mammalian cells. Nat Biotechnol. 2001 19(12):1173-1176.

38. Rao Y, Haucke V. Membrane shaping by the Bin/amphiphysin/Rvs (BAR) domain protein superfamily. Cell Mol Life Sci. 2011 68(24):3983-3993.

39. Kessels MM, Qualmann B. Different functional modes of BAR domain proteins in formation and plasticity of mammalian postsynapses. J Cell Sci. 2015 128(17):3177-3185.

40. Sánchez-Barrena MJ, Vallis $Y$, Clatworthy $M R$, et al. Bin2 is a membrane sculpting N-BAR protein that influences leucocyte podosomes, motility and phagocytosis. PLoS One. 2012 7(12):e52401.

41. Volz J, Kusch C, Beck S, Popp M, Vögtle T, Meub M, Scheller I, Heil HS, Preu J, Schuhmann MK, Hemmen K, Premsler T, Sickmann A, Heinze KG, Stegner D, Stoll G, Braun A, Sauer M, Nieswandt B. BIN2 orchestrates platelet calcium signaling in thrombosis and thrombo-inflammation. J Clin Invest. 2020 130(11):6064-6079.

42. Meyuhas O. Ribosomal Protein S6 Phosphorylation: Four Decades of Research. Int Rev Cell Mol Biol. 2015 320:41-73.

43. Hutchinson JA, Shanware NP, Chang H, Tibbetts RS. Regulation of ribosomal protein $\mathrm{S} 6$ phosphorylation by casein kinase 1 and protein phosphatase 1. J Biol Chem. 2011 286(10):8688-8696.

44. Ang Z, Koean RAG, Er JZ, Lee LT, Tam JKC, Guo H, Ding JL. Novel AU-rich proximal UTR sequences (APS) enhance CXCL8 synthesis upon the induction of rpS6 phosphorylation. PLoS Genet. 2019 15(4):e1008077. 
45. Reddy P, Adhikari D, Zheng W, Liang S, Hämäläinen T, Tohonen V, Ogawa W, Noda T, Volarevic S, Huhtaniemi I, Liu K. PDK1 signaling in oocytes controls reproductive aging and lifespan by manipulating the survival of PMFs. Hum Mol Genet. 2009. 18(15):2813-2824.

46. Vozenilek AE, Vetkoetter M, Green JM, Shen X, Traylor JG, Klein RL, Orr AW, Woolard MD, Krzywanski DM.

Absence of Nicotinamide Nucleotide Transhydrogenase in C57BL/6J Mice Exacerbates Experimental Atherosclerosis. J Vasc Res. 2018 55(2):98-110.

47. Son MJ, Kwon Y, Son T, Cho YS.

Restoration of Mitochondrial NAD(+) Levels Delays Stem Cell Senescence and Facilitates Reprogramming of Aged Somatic Cells. Stem Cells. 2016 34(12):2840-2851 . 


\section{FIGURES WITH LEGENDS}

Figure 1. Bin2 is rich in oocytes and essential for primordial follicle activation.

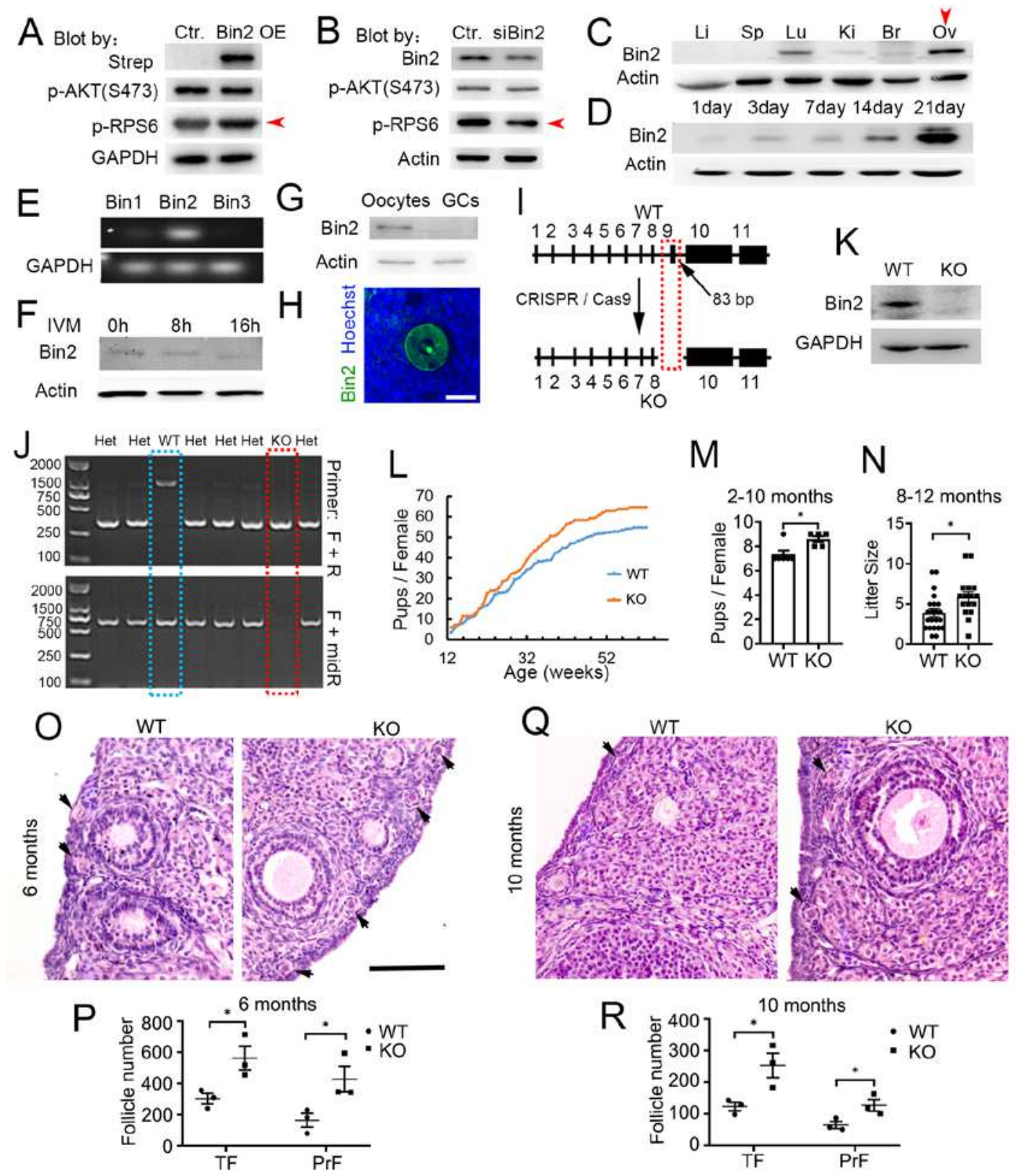

A. Western blot showed that Bin2 overexpression in human epithelial ovarian cancer (EOC) cells A2780 significantly up-regulated p-RPS6 (red arrow) of mTOR pathway, while not altering p-Akt. B. Western blot showed that Bin2 knockdown in A2780 significantly down-regulated p-RPS6 (red arrow) of mTOR pathway, while not altering p-Akt. C. Western blot showed that Bin2 protein was the richest in ovarian tissue (arrow pointed), compared with other major tissues. Li, liver; Br, brain; Ov, ovary; Sp, spleen; Ki, kidney; Lu, lung. D. Bin2 protein level sharply increased as the initial follicle recruitment 
happened (around PND 21). PND, post-natal-day. E. RT-PCR in oocytes showed that the Bin2 mRNA level was much higher than the levels of another two Bin family members, Bin1 and Bin3. F. Western blot showed that Bin2 level remained constant during meiosis. $\mathrm{G}$ and $\mathrm{H}$. Western blot and immunofluorescence showed that Bin2 protein was more dominant in oocytes than in granulosa cells. I and J. We used cas 9 technique to delete the entire exon 9 (83 bases, red rectangle-circled) of Bin2 genome to obtain Bin2 global knockout $(\mathrm{KO})$ mice, western blot showed that Bin2 protein completely disappeared in the Bin2-knockout ovaries. K. Genotypying of Bin2-KO mice through PCR and DNA gel electrophoresis. Blue rectangle indicates WT mouse, red rectangle indicates Bin2-KO mouse. L-N. 60-week-long fertility assay showed that in Bin2-KO group, the cumulative litter number per mouse significantly increased $(L)$; the average litter numbers per female from 2 to 10 months significantly increased $(M)$; and the average litter size (pups/litter) from 8 to 12 months also significantly increased (N). O-R. Continuous follicle count showed that at 6 months old ( $O$ and $P$ ) or 10 months old $(Q$ and $R)$, total follicle numbers and primordial follicle numbers significantly increased in Bin2-KO ovaries. Scale bar for $\mathrm{H}$, $50 \mu \mathrm{m}$; scale bar for $\mathrm{O}$ and $\mathrm{Q}, 100 \mu \mathrm{m}$. * indicates $\mathrm{p}<0.05$.

\section{Figure 2. Bin2 knockout specifically down-regulated the phosphorylation of RPS6 of mTOR pathway.}
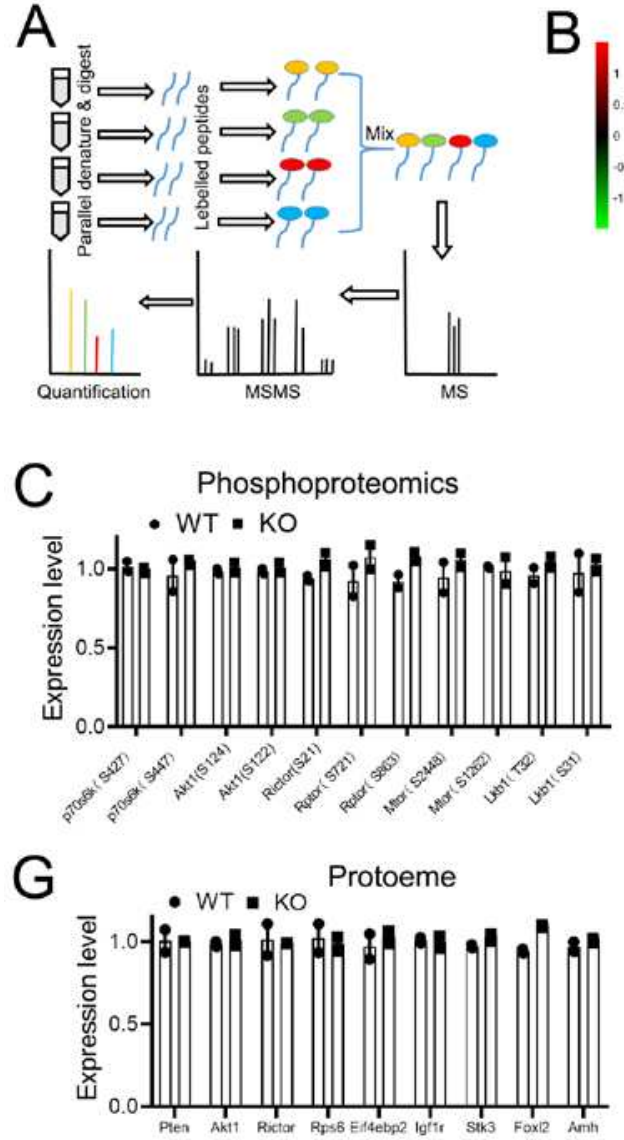

B
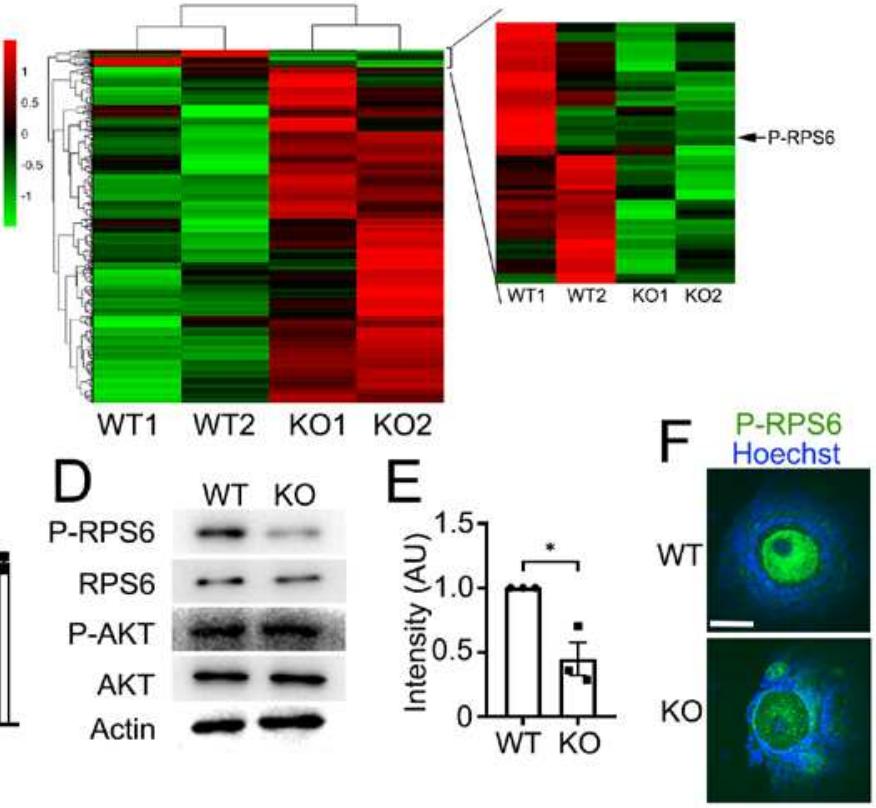

RNAseq

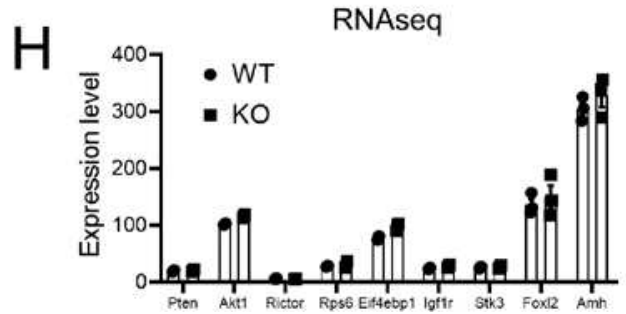


A. We used TMT-labeled quantitative phosphomics to systematically identify differentially phosphorylated proteins $¥ 1.2$ fold or $\leq 0.833$ fold) between control and Bin2-KO 3-week-old ovaries. B and C. We found that among mTOR pathway proteins, only RPS6 had significantly decreased phosphorylation. D-F Both western blot and immunofluorescence showed that among mTOR pathway proteins, only RPS6 had significantly decreased phosphorylation in Bin2-KO ovaries. G. TMT-labeled quantitative gross proteomics showed that the gross protein level of each mTOR pathway component was unaltered. $\mathrm{H}$. RNA sequencing showed that the mRNA level of each mTOR pathway component (pTEN, Akt1, Rictor, Rps6, Eif4ebp1, IGF1R, Stk3, Foxl2, and Amh) was unaltered. Scale bar, $50 \mu \mathrm{m} .{ }^{*}$ indicates $p<0.05$.

\section{Figure 3. Phosphorylation at Thr423 \& Ser424 activates Bin2.}

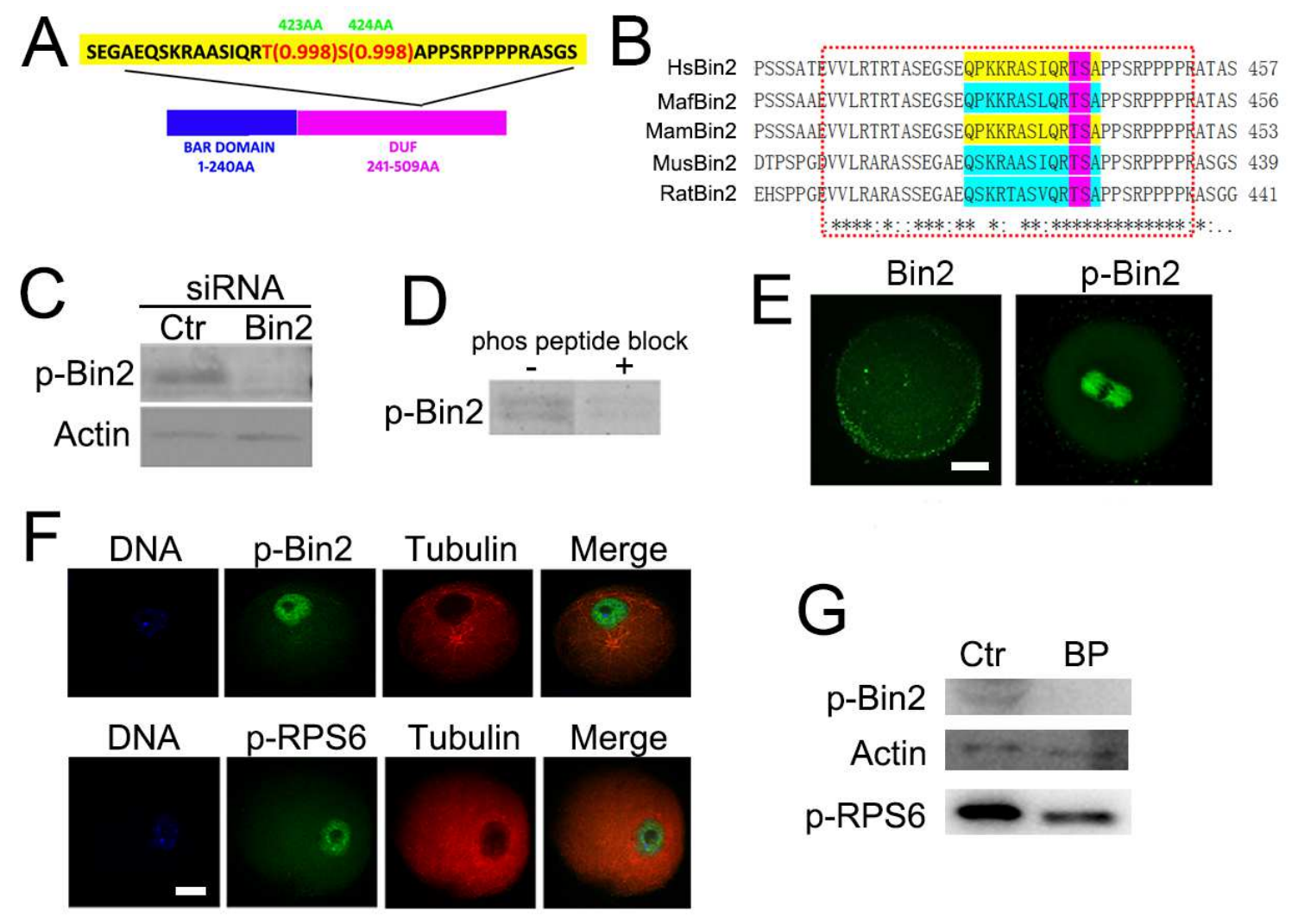

A. We identified several phosphorylation sites (Supplementary table 1); among these, Thr423 \& Ser424 were in the c-terminal domain of unknown function (DUF domain). B. Thr423 \& Ser424 and the adjacent residues are highly conserved between humans, rats, mice, rhesus monkeys, and cynomolgus macaques. $C$ and D. We hypothesized that Thr423 \& Ser424 are the key residues for the activation and translocation of Bin2. We generated a phosphorylation antibody using a core phosph peptide "QSKRAASIQR(pThr)(pSer)A" and verified its specificity through Bin2 siRNA and corresponding non-phosph peptide "QSKRAASIQRTSA." E. Immunofluorescence 
showed that Bin2 was enriched on oocyte membrane, while $p$-Bin2 was enriched within spindles during meiosis. F. Immunofluorescence showed that p-Bin2 was enriched within the nucleus of GV oocytes, similar to p-RPS6. G. We injected the non-phosph peptide "QSKRAASIQRTSA" into GV oocytes and found that the p-Bin2 level significantly decreased, while the p-RPS6 level also significantly decreased. Scale bar, $20 \mu \mathrm{m}$.

\section{Figure 4. In-vitro characterization of Bin2 phosphorylation activity on RPS6.}

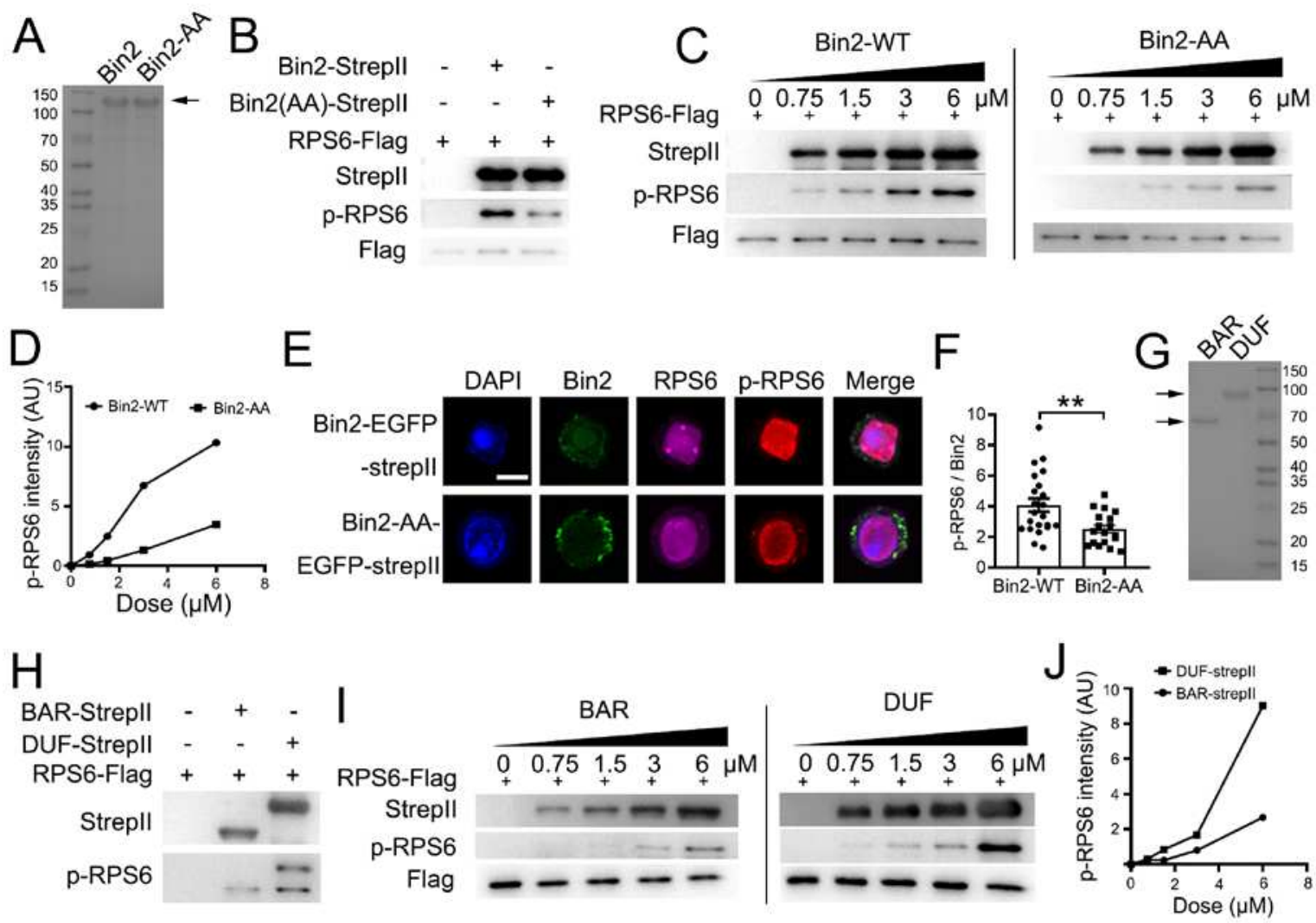

A. We used the Bac-to-bac system to express full-length wild-type Bin2 (Bin2-WT) and a Bin2-AA mutant (Thr423 \& Ser424 to Ala423 \& Ala 424). SDS-PAGE and commassie staining showed that both purified proteins had good purity and expected size. B. In-vitro phosphorylation and western blot showed that Bin2-WT can phosphorylate RPS6 more than an equal amount of Bin2-AA. C. In-vitro, dose-dependent phosphorylation assay and western blot showed that Bin2-WT could phosphorylate RPS6 much more strongly than Bin2-AA. D. Quantification of C. E. Immunostaining on bacularvirus-infected sf9 cells showed that Bin2-WT localized both on the membrane and within the cytoplasm, while Bin2-AA almost exclusively localized on the membrane. Accordingly, cytoplasmic p-RPS6 intensity in Bin2-WT bacularvirus-infected infected sf9 cells was significantly higher than in Bin2-AA bacularvirus-infected sf9 cells. F. Quantification of E. G. We expressed and purified two different motifs of Bin2, BAR (1-240 AA) and DUF (domain of 
unknown function, 241-505 AA); SDS-PAGE and commassie staining showed that both purified proteins had good purity and expected size. $\mathrm{H}$. In-vitro phosphorylation and western blot showed that Bin2-DUF can phosphorylate RPS6 more than an equal amount of Bin2-BAR. I. In-vitro dose-dependent phosphorylation assay and western blot showed that Bin2-DUF could phosphorylate RPS6 much more strongly than Bin2-BAR. J. Quantification of I. Scale bar, $10 \mu \mathrm{m} .{ }^{* *}$ indicates $p<0.01$.

\section{Figure 5. Bin2 knockout promotes NNT translation through down-regulating p-RPS6.}
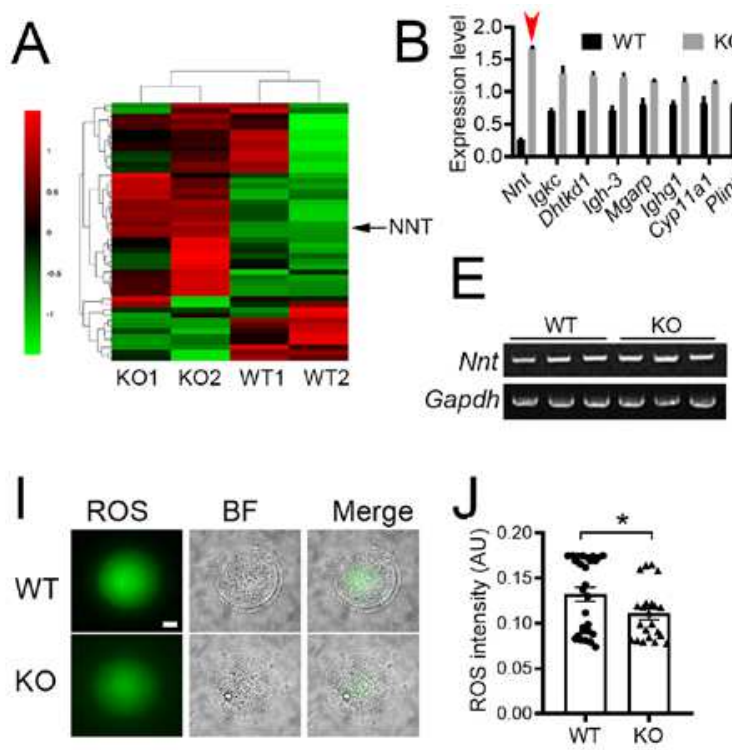

M

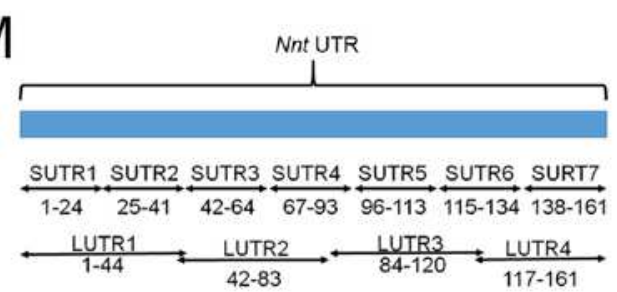

$\mathrm{P}$

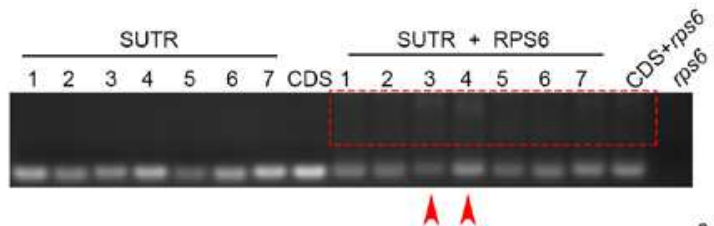

$\mathrm{R}$

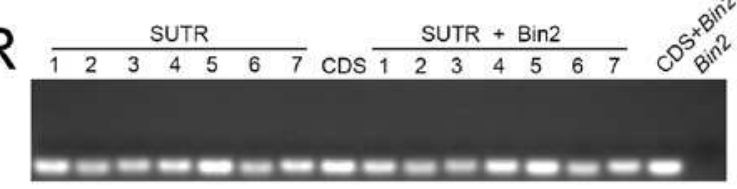

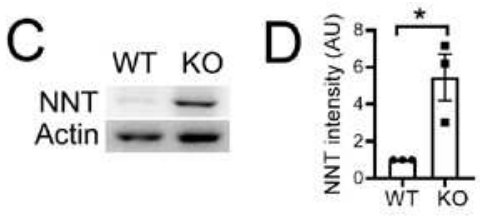
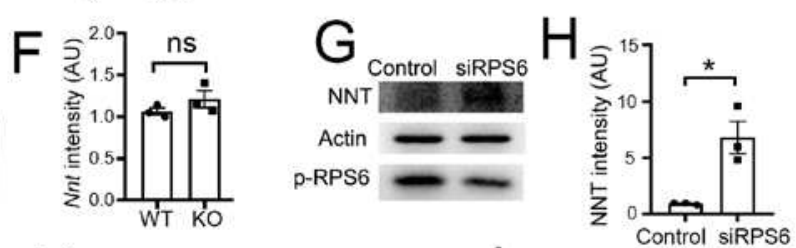

$\mathrm{L}$
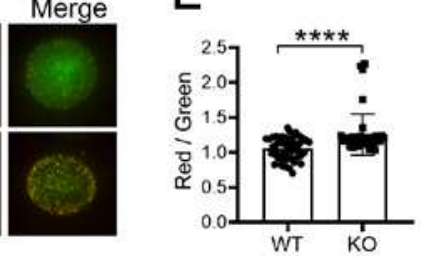

$\mathrm{N}$
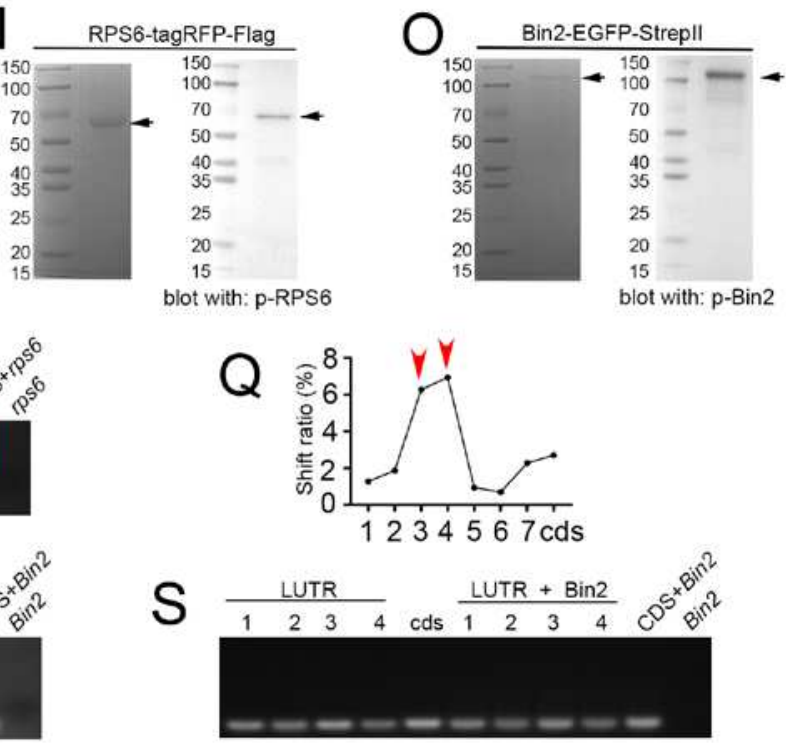

$A$ and B. We used TMT-labeled quantitative proteomics to systematically identify differentially expressed gross proteins between control and Bin2-knockout, 3-week-old ovaries ( $\geq 1.2$ fold or $\leq 0.833$ fold). Among the differentially expressed gross proteins, 
nicotinamide nucleotide transhydrogenase (NNT) was most up-regulated (> 6 fold, red-arrow pointed). C and D. Western blot and quantification verified that the NNT protein level in the Bin2-knockout group increased more than 6-fold compared to control. E and F. RT-PCR and quantification showed that Bin2 knockout did not affect the mRNA level of NNT. G and H. Western blot and quantification showed that the NNT protein level in the RPS6-knockdown group significantly increased. I and J. Live dye staining and quantification showed that the ROS level significantly decreased in Bin2-knockout oocytes. $\mathrm{K}$ and L. JC-1 staining and quantification showed that the mitochondria membrane potential significantly increased in Bin2-knockout oocytes. M. We attempted to address whether $p$-Bin2 or p-RPS6 bind the translation-regulating regions of NNT to inhibit its translation. We subdivided NNT 5' UTR into seven shorter or four longer regions and in-vitro transcribed single-strand RNA of these regions. $\mathrm{N}$ and O. SDS-PAGE, commassie staining, and western blot showed that RPS6 $(\mathrm{N})$ and Bin2 $(\mathrm{O})$ proteins had phosphorylated portions. P and Q. Protein-RNA UV crosslinking and gel shift assay showed that p-RPS6 prefer to bind short region three and four, from 42 to 95 bp of NNT 5' UTR (red-arrow pointed), instead of other UTR regions or CDS region. Q. Quantification of P. R and S. Protein-RNA UV crosslinking and gel shift assay showed that $p$-Bin2 did not bind any shorter or longer regions. Scale bar, $20 \mu \mathrm{m}$. ${ }^{*}$ indicates $p<0.05$, *** indicates $p<0.0001$. 


\section{Figure 6. Inhibition of Bin2 phosphorylation in vivo prolongs ovarian lifespan of aging mice.}

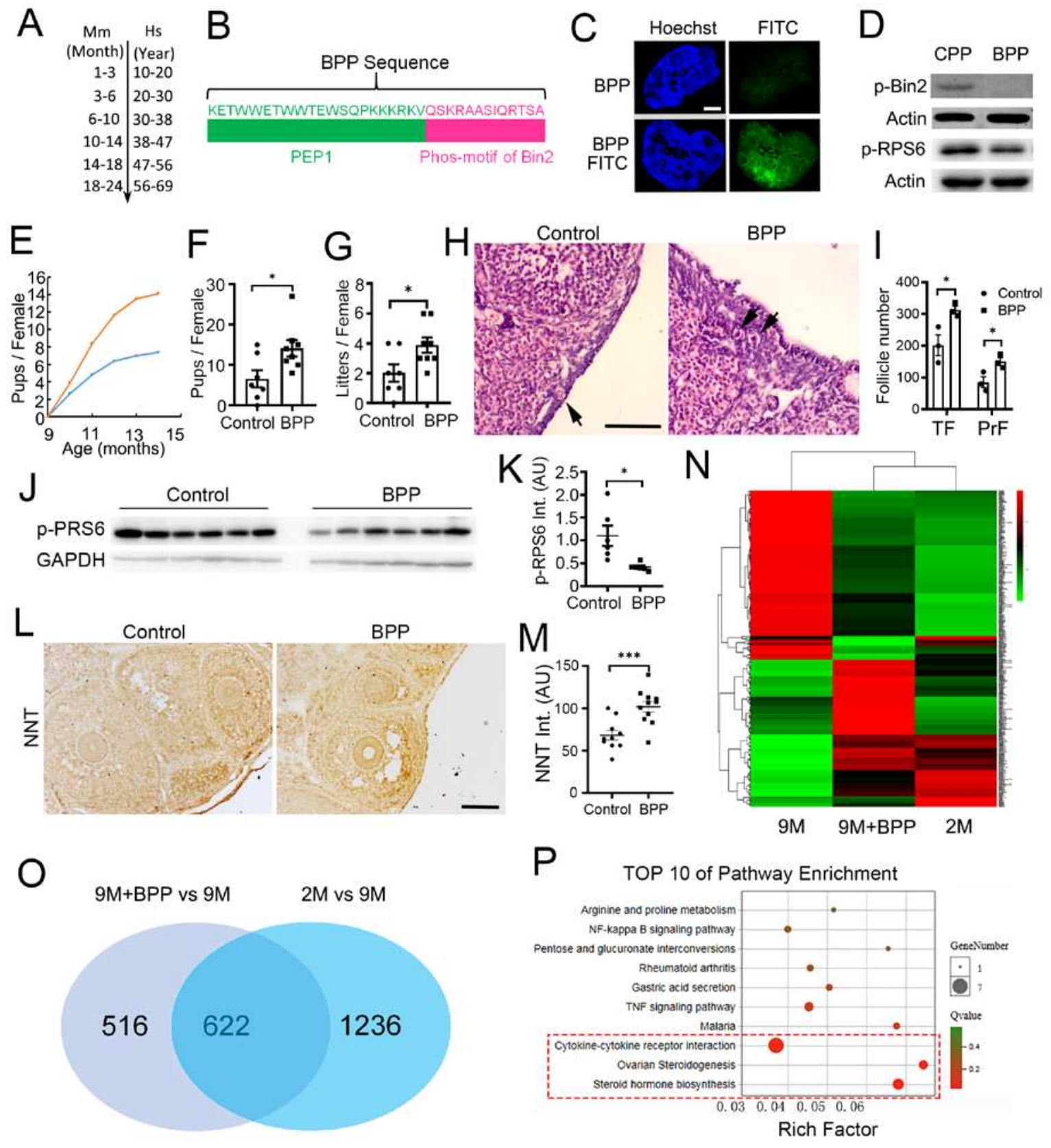

A. Analogical time axis between mouse and human. We planned to use 8-month-old aging mice as models to mimic 35-year-old women. $B$. To facilitate the permeation of this peptide in vivo, we fused PEP1, the known cell-penetrating peptide, to the non-phosphor Bin2 peptide "QSKRAASIQRTSA" and renamed it as BPP (Bin2-penetrating peptide). C and D. We firstly verified that FITC-labeled BPP can efficiently enter ovaries three days after injection (C), and both p-Bin2 and p-RPS6 significantly decreased within ovaries (D). E-G. After a 3-week-long BPP injection cycle (once a day) in 8-month-old aging mice, fertility assay from 36 weeks old to 63 weeks old showed that both the average number 
of cumulative pups per female ( $E$ and $F$ ) and average litters per female ( $E$ and $G$ ) significantly increased. $\mathrm{H}$ and $\mathrm{I}$. Continuous follicle counting showed that both total follicles and PMFs significantly increased in the BPP-treated group. $\mathrm{J}$ and $\mathrm{K}$. Western blot and quantification showed that p-RPS6 significantly decreased in the BPP-treated group. L and M. Immunostaining and quantification showed that the NNT level within the oocytes significantly increased in the BPP-treated group. $\mathrm{N}$ and $\mathrm{O}$. We examined how well BPP could recover ovarian function in the aging mice at the transcriptional level. RNA sequencing showed that at the fold threshold of $\mid \log 2$ (2-month/9-month) $\mid \geq 2$, there were 1858 DEGs (differentially-expressed genes) between 2-month ovaries and 9-month ovaries; at the fold threshold of $\mid \log 2(9$-month-BPP/9-month) $\mid \geq 2$, there were 1138 DEGs (differentially-expressed genes) between 9-month-BPP ovaries and 9-month ovaries; and between these two clusters of DEGs, 622 DEGs overlapped. P. KEGG analysis of functionally-annotated DEGs showed that multiple follicle survival, oocyte quality, or oocyte steriodgenesis-related pathways are among the top 10 enriched signaling pathways. Scale bar for B, $200 \mu \mathrm{m}$; scale bar for H and L, $100 \mu \mathrm{m}$. * indicates $\mathrm{p}<0.05$.

\section{Figure 7. Inhibition of Bin2 phosphorylation in vivo protects ovaries from chemotherapy-induced premature ovarian failure.}

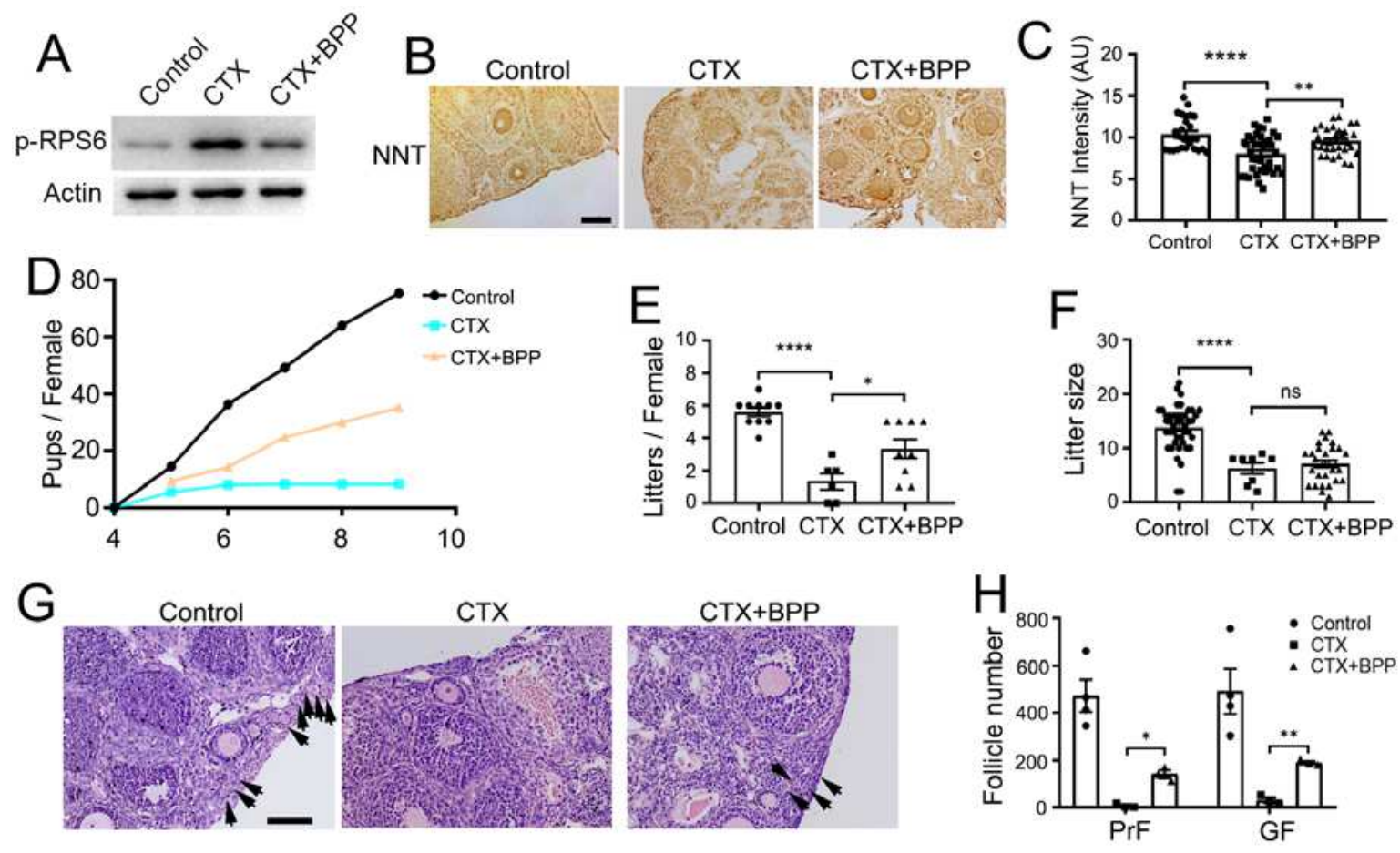

A. Western blot showed that a harsh, three-week-long cyclophosphamide (CTX) daily treatment significantly elevated p-RPS6, while BPP treatment reduced the p-RPS6 level close to control level. B and C. Immunostaining and quantification showed that CTX 
treatment significantly decreased the oocyte NNT level, while BPP injection recovered the NNT level close to control. D-F. Fertility assay from 4 months old to 9 months old showed that CTX treatment caused almost completed infertility, while BPP significantly recovered fertility (although still less than control). $\mathrm{G}$ and $\mathrm{H}$. Continuous follicle counting showed that CTX treatment sharply decreased follicle numbers at each stage, while BPP could significantly increased follicle numbers at each stage (although still less than control). Scale bar, $100 \mu \mathrm{m} .{ }^{*}$ indicates $p<0.05,{ }^{* *}$ indicates $p<0.01,{ }^{* * * *}$ indicates $p<$ 0.0001 .

\section{Figure 8. Bin2 working model.}

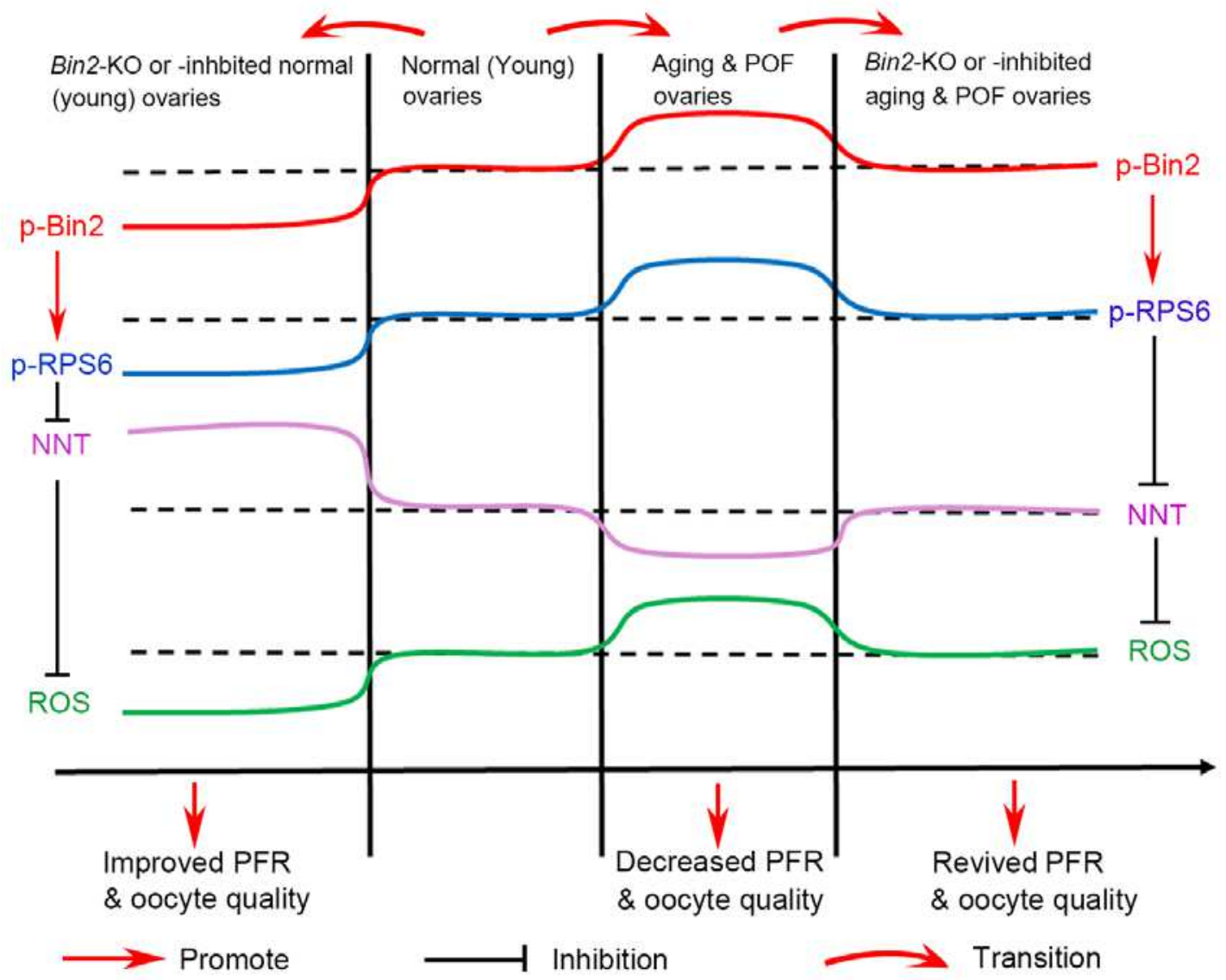

In this model, the correlations of primordial follicle and oocyte quality with the level of p-Bin2, p-RPS6, NNT, and ROS are shown under four conditions. Two adjacent conditions are isolated by a vertical axis representing the suppositional protein levels. From left to right, Bin2-KO or -inhibited normal (young) ovaries, normal (young) ovaries, aging \& POF ovaries, and Bin2-KO or -inhibited aging \& POF ovaries. The red curved arrows on the top indicated the transitions from one condition to the other. Four solid colored horizontal lines (red for p-Bin2, blue for p-RPS6, magenta for NNT, and black for ROS ) connected by dotted line indicate the suppositional normal level of these four 
substances. Upon transition from normal (young) ovaries to Bin2-KO or -inhibited normal (young) ovaries, p-Bin2 is downregulated and conduced to p-RPS6 downregulation, NNT upregulation, and ROS downregulation, which eventually improve PFR and oocyte quality. Upon transition from normal (young) ovaries to aging \& POF ovaries, p-Bin2 is upregulated and conduced to p-RPS6 upregulation, NNT downregulation, and ROS upregulation, which eventually decrease PFR and oocyte quality. Upon transition from aging \& POF ovaries to Bin2-KO or -inhibited aging \& POF ovaries, higher p-Bin2 is downregulated close to normal level and conduced to higher p-RPS6 downregulated close to normal level, lower NNT upregulated close to normal level, and higher ROS downregulated close to normal level, which eventually revive PFR and oocyte quality.

\section{MATERIALS AND METHODS}

\section{Knockout Mice}

Animal experimental procedures in our study were all approved by the Animal Ethics Committee of Nanjing Medical University (approval no. IACUC-1809011), and all mice were housed under standard specific pathogen free (SPF) conditions of ACF.

The global Bin2-knockout C57/B6 mice were made in the Animal Core Facility (ACF) of the State Key Lab of Stem Cell and Reproductive Biology (SKLSCRB) of the Institute of Zoology, Chinese Academy of Sciences, and then transferred to the ACF of the State Key Lab of Reproductive Medicine (SKLRM) of Nanjing Medical University and re-cleansed. CRISPR/Cas9 technology was used. We planned to delete the whole exon 9, which is shared by three isoforms of Bin2. For this, we designed two 20-base, gene-complementary oligos of the sgRNA within the introns upstream and downstream of exon9, respectively (Supplementary Table 2.1). Each of these four oligos were inserted into pUC57-T7-gRNA. The sgRNA was produced by MEGAshortscript ${ }^{\mathrm{TM}}$ Kit (Thermo Fisher Scientific, Waltham, MA, USA) using linearized pUC57-T7-Bin2 gRNA as a template and purified by MEGAclearTM Kit (Thermo Fisher Scientific). The cas9 mRNA was first produced by mMessage 
mMachine T7 kit (Thermo Fisher) using linearized pST1374-N-NLS-flag-linker-Cas9 as a template. Then the mRNA was poly A-tailed (to increase mRNA stability) using poly A-tailing kit (Thermo Fisher) and purified by RNeasy Micro kit (Qiagen, Dusseldorf, Germany). SgRNA and cas9 mRNA were sent to ACF of SKLSCRB; pronuclear microinjection, embryo transfer, and mouse parturition was accomplished by professional staff in the ACF. Genotyping of Bin2-KO mice (Fig. 1I) was done by PCR and DNA sequencing. Forward primer is 5'-GATTCTAAGCTGCTTCCACATTAC-3'; reverse primer is 5'-TGGCTGGTCTCAGATTTATGAC-3'. The genotyping PCR program was as follows: $94{ }^{\circ} \mathrm{C}$ for 5 min, 35 cycles of melting at $94^{\circ} \mathrm{C}$ for $30 \mathrm{~s}$, annealing at $57^{\circ} \mathrm{C}$ for $15 \mathrm{~s}$, and extension at $72{ }^{\circ} \mathrm{C}$ for $30 \mathrm{~s}$, with additional extension at $72{ }^{\circ} \mathrm{C}$ for 5 minutes at the end.

For all fertility assay, WT mating male mice were monthly rotated between cages according to a random allocation table (supplementary dataset 1 ).

\section{Model Mice}

8-month-old C57/B6 retiring mice (equal to 34.5-year-old human female) were bought from ACF and randomly divided into two groups: Control group, treated with $0.5 \mathrm{mg} / \mathrm{kg}$ Control peptide, PEP1 (KETWWETWWTEWSQPKKKRKV); and BPP $\begin{array}{llllll}\text { group, } & \text { treated } & \text { with } & 0.5 & \mathrm{mg} / \mathrm{kg} & \text { BPP }\end{array}$ (KETWWETWWTEWSQPKKKRKVQSKRAASIQRTSA) once daily for three weeks. BPP and PEP1 were synthesized by Shanghai Bootech BioScience \& Technology Company (Shanghai, China). The treated mice were subjected to diverse assays (Fig. 6 ) at 9 months old (equal to 36.5-year-old human female).

2-month-old ICR mice (equal to 16-year-old human female) were bought from ACF and randomly divided into three groups: Control group, treated with $0.5 \mathrm{mg} / \mathrm{kg}$ PEP1; CTX-only group, treated with $75 \mathrm{mg} / \mathrm{kg}$ cyclophosphamide (CTX, Cayman, Ann Arbor, Michigan, USA) once daily for three weeks; and CTX + BPP group, treated with $75 \mathrm{mg} / \mathrm{kg} \mathrm{CTX}$ and $0.5 \mathrm{mg} / \mathrm{kg}$ BPP once daily for three weeks. The treated mice were subjected to diverse assays (Fig. 7) at 3 months old (equal to 20-year-old human female). 


\section{Antibodies}

Primary antibodies: rabbit anti-Phosphorylation AKT (T308) (Cat\#: 13038; Cell Signaling Technology, Danvers, MA, USA); NNT Polyclonal antibody (Cat\#: 13442-2-AP; Proteintech, Rosemont, IL, USA); RPS6 (L234) polyclonal antibody (Cat\#: BS1592; Bioworld, Bloomington, MN, USA); mouse anti strep II-Tag mAb (Cat\#: AE066, ABclonal, Wuhan, Hubei, China); Phospho-Akt (Ser473) (D9E) XP® Rabbit mAb (Cat\#: 4060; Cell Signaling Technology); GAPDH (D16H11) XP® Rabbit mAb (Cat\#: 5174; Cell Signaling Technology); $\beta$-Actin (13E5) Rabbit mAb (Cat\#: 4970; Cell Signaling Technology); Acetyl-a-Tubulin (Lys40) (D20G3) (Cat\#: 5335; Cell Signaling Technology); Phospho-S6 Ribosomal Protein (Ser235/236) Antibody (Cat\#:2211, Cell Signaling Technology); Akt Antibody (Cat\#: 9272; Cell Signaling Technology); and Mouse anti DDDDK-Tag mAb (Cat\#: AE005, ABclonal).

Secondary antibodies: Horseradish peroxidase (HRP)-conjugated rabbit anti-goat IgG and HRP-conjugated goat anti-mouse IgG were purchased from Vazyme (Nanjing, Jiangsu, China). Rhodamine(TRITC)-conjugated donkey anti-rabbit IgG (Code: 711-025-152), Cy5-conjugated donkey anti-mouse IgG (Code: 1715-175-150), and Cy2-conjugated donkey anti-rabbit IgG (Code: 711-225-152) were purchased from Jackson ImmunoResearch Laboratory (West Grove, PA, USA).

\section{Oocyte Collection and In Vitro Culture}

Fully-grown GV oocytes were collected from three-week-old female mice. Oocytes were released by puncturing follicles with a sterile syringe needle in a MEM+ medium (0.01 mM EDTA, $0.23 \mathrm{mM}$ Na-pyruvate, $0.2 \mathrm{mM}$ penicillin/streptomycin and 3 $\mathrm{mg} / \mathrm{ml}$ BSA in MEM). After washing away the cumulus cells from the cumulus-oocyte complexes, oocytes were cultured in $100 \mu$ l mini-drops of MEM+ containing $20 \%$ fetal bovine serum (FBS) (Thermo Fisher) covered with mineral oil at $37.0^{\circ} \mathrm{C}$ in an incubator with $5 \% \mathrm{O}_{2}, 5 \% \mathrm{CO}_{2}$, and a humidified atmosphere.

\section{Cell Culture and Plasmid Transfection}

Human epithelial ovarian cancer (EOC) cells, A2780, were from ECACC (Cat No.: 93112519) and sold by Procell Life Science \& Technology Co. (Wuhan, Hubei, China). 
The cells were cultured in DMEM with 10\% FBS. For plasmid transfections, cells were grown at $50 \%-70 \%$ confluence, and were transfected with Lipofectamine ${ }^{\mathrm{TM}} 3000$ Transfection Reagent (Thermo Fisher) according to the manufacturer's protocol.

\section{Immunofluorescence Staining of Oocytes and Image Taking}

Oocytes were permeabilized with $0.5 \%$ Triton X-100/PHEM (60 mM PIPES, 25 $\mathrm{mM}$ Hepes, $\mathrm{pH}$ 6.9, $10 \mathrm{mM}$ EGTA, $8 \mathrm{mM} \mathrm{MgSO}_{4}$ ) for five minutes, and then were fixed in 3.7\% FPA in PHEM for 20 minutes at room temperature. After being washed with PBS/0.05\% PVP (polyvinylpyrrolidone) three times at 10 minutes each, oocytes were blocked in blocking buffer (100 mM glycine and 1\% BSA in PBS) for one hour at room temperature. Primary antibodies were then diluted in blocking buffer and oocytes were incubated in it overnight at $4.0^{\circ} \mathrm{C}$. Fluorescent secondary antibodies (Jackson ImmunoResearch Laboratories) were used at $7.5 \mu \mathrm{g} / \mathrm{ml}$. DNA was stained with $0.3 \mu \mathrm{g} / \mathrm{ml}$ Hoechst 33258. Next, buffers were were slowly and gentely removed from around the oocytes, which immobilized the oocytes on the slide; then a drop (about 5-10 $\mu$ l) of anti-fade solution ( $0.25 \% \mathrm{n}$-propyl gallate and $90 \%$ glycerol in PBS) was mounted onto the oocytes and oocytes were covered by a coverglass. To avoid the deformation of the oocytes, a double-stick tap was pre-placed between the slides and coverslips. Specimens were imaged with IQ2 on an Andor Revolution spinning-disk confocal system (Andor Technology PLC, Belfast, UK) mounted on an inverted TiE microscope (Nikon, Japan) with a $60 \times, 1.4 \mathrm{NA}$ objective and captured with cold CCD camera (Andor). Most images are displayed as maximum intensity projections of the captured $z$ stacks.

\section{Ovarian Hematoxylin-Eosin Staining and Ovary Follicle Counting}

Ovaries were obtained, washed and fixed in $10 \%$ buffered formalin or $4 \%$ PFA overnight, embedded in paraffin, continuously sectioned at $5 \mu \mathrm{m}$ thickness, then stained with hematoxylin and eosin. The follicle stages were classified according to Pederson's standard. Only follicles with visible nuclei were counted. In brief, an oocyte surrounded by a single layer of flattened or cubical granulosa cell was defined as a primordial or primary follicle; an oocyte surrounded by more than one layer of 
cuboidal granulosa cells without visible antrum was defined as a secondary follicle; and a follicle possessing a clearly antral space containing follicular fluid was defined as antral follicle. The number of follicles counted per ovary was used for statistical analysis.

\section{Identification of Bin2 Phosphorylation Site and Generation of p-Bin2-specific Antibody}

Generally, the phosphorylated part of a protein is about $0.01-0.1 \%$, making it almost impossible to use oocytes for identification of phosphorylation sites. Therefore, we used NIH3T3 cells instead, then generated a phospho-specific antibody and verify the antibody in oocytes. We used five IP reactions, each of which employed $2.5 \times 10^{6}$ NIH3T3 cells, $30 \mu \mathrm{l}$ protein $\mathrm{A} / \mathrm{G}$ beads and $2 \mu \mathrm{g}$ Bin2 antibody. Then the immuno-complexed beads were eluted by $0.2 \mathrm{M}$ glycine $(\mathrm{pH} 2.7)$, and the phosphorylated portion of the immuno-complex was enriched by Pierce ${ }^{\mathrm{TM}} \mathrm{TiO}_{2}$ Phosphopeptide Enrichment and Clean-up Kit (Thermo Scientific, Rockford, IL, USA) and sent to Testing \& Analysis Center, Nanjing Medical University for LC-MS (liquid chromatograph-mass spectrometer). We chose Thr423 \& Ser424 (phosphorylation possibility $99.8 \%$ ), and the whole process of antibody production and purification was accomplished by Zoonbio Biotechnology (Nanjing, Jiangsu, China). A short phospho peptide QSKRAASIQR(pThr)(pSer)AC ("C" at the C-term is an extra residue for conjugation) was synthesized and injected into rabbits for the serum production. The phospho-specific antibody was purified from the serum through a column filled with phospho peptide-conjugated resin and then absorbed through a column filled with non-phospho peptide (QSKRAASIQRTSA)-conjugated resin to remove any residual non-phospho-specific antibody.

\section{Expression and Purification of Recombinant Bin2 and RPS6 Proteins with Bac-to-bac System}

EGFP-tagged Bin2-WT (wild type), Bin2-AA (T423A, S424A), Bin2-BAR (BAR domain of Bin2), Bin2-DUF (DUF domain of Bin2), and TagRFP-tagged rps6 proteins were cloned and expressed through a Bac-to-bac system (Thermo Fisher). In brief, 
the corresponding sequences (Supple. Table 2.2) were cloned into pFastBacHTA (Thermo Fisher) and then transformed into DH10Bac E. coli cells. The bacmid was isolated from DH10Bac E. coli (Vazyme) with QIAfilter Plasmid Purification Kit (Qiagen) and then transfected into Sf9 cells (Cat No.: ATCC $\AA$ CRL-3357 TM, sold by Genetimes ExCell Technology, Shanghai, China) with Cellfectin® II Transfection reagent (Thermo Fisher) in the static upper plane of an orbital shaker (Shanghai Zhichu Instrument Co. Shanghai, China) at $27^{\circ} \mathrm{C}$. The virus-containing supernatant was used to infect fresh Sf9 cells for 72 hours, and the procedure was repeated twice to amplify the baculovirus. $20 \mu \mathrm{l}$ of the third cycle supernatant was added to Sf9 cells in $250 \mathrm{ml}$ SFM900-II medium (Thermo Fisher) with 5\% FBS to express proteins in the lower bed of an orbital shaker at $27{ }^{\circ} \mathrm{C}, 200 \mathrm{rpm}$. Next, infected cells were resuspended in a lysis buffer (containing $50 \mathrm{mM}$ Tris, 10\% sucrose, $50 \mu \mathrm{M}$ ATP, $1 \mathrm{mM}$ PMSF, $5 \mathrm{mM}$ DTT, 1\% NP40, $10 \mathrm{mM}$ imidazole, 1x protease inhibitor and phosphatase inhibitor, $\mathrm{PH} 7.0$ by $\mathrm{HCl}$ ) and lysed with a high pressure cell disrupter (Union Biotech, Shanghai, China), centrifuged, and the resulting supernatant was incubated with $1 \mathrm{ml} \mathrm{Ni-NTA} \mathrm{Superflow} \mathrm{resin} \mathrm{(Qiagen)} \mathrm{at} 4{ }^{\circ} \mathrm{C}$ for 1 hour. The resin was then transferred into a $5 \mathrm{ml}$ chromatography column (Biocomma Co., Shenzhen, Guangdong, China), washed with four column volumes of wash buffer (40 mM imidazole in resuspension buffer without PMSF), and eluted with $500 \mathrm{mM}$ imidazole in resuspension buffer without PMSF. The eluted protein was concentrated by a size-exclusion spin column and exchanged into BRB80 (80 mM HEPES, $1 \mathrm{mM} \mathrm{MgCl}$, $1 \mathrm{mM}$ EGTA, pH 6.8 by $\mathrm{KOH}$ ) with $10 \%$ glycerol, $50 \mu \mathrm{M}$ ATP and $5 \mathrm{mM}$ DTT. The protein was aliquoted and kept in a $-80^{\circ}$ freezer for future use.

\section{Expression and Purification of Recombinant RPS6 Proteins in E. coli}

For non-phosphorylated form of RPS6, recombined RPS6-tagRFP-flag sequence was cloned into pRSETB (primers are in Supplementary Table 2.3) and transformed into BL21-DE competent E. coli (Vazyme). The transformed E. coli was grown in $4 \times 1$ $\mathrm{L} \mathrm{LB}+$ medium in an orbital shaker (Shanghai Zhichu Instrument Co.) at $37^{\circ} \mathrm{C}, 220$ 
rpm until OD600 reached 1.0. Next, $0.2 \mathrm{mM}$ IPTG was added into the medium to induce the recombined RPS6 expression overnight at $16{ }^{\circ} \mathrm{C}, 220 \mathrm{rpm}$. The next morning, the E.coli was spun down, washed once with cold PBS, lysed, and purified as descried above.

\section{In Vitro Phosphorylation Assays}

BRB80 with 10\% glycerol, $1 \mathrm{mM}$ ATP and $5 \mathrm{mM}$ DTT was used for the in vitro phosphorylation assays. Appropriate amounts of Bin2 proteins and RPS6 were incubated at RT for 20 minutes. Finally, the reaction was subjected to western blot.

\section{TMT-Labeling Quantitative Proteomics and Phosphomics}

About two hundred three-week-old ovaries per repeat (about $200 \mathrm{mg}$ ), two repeats for WT and Bin2-KO respectively, were sent to Hangzhou Jingjie Co. (Hangzhou, China). In brief, ovaries were cracked and the supernatant was digested by trypsin into peptides. Then the peptides from individual samples were isobaric-mass tagged by $\mathrm{TMT}^{6}-126,127,128$, and 129 , respectively, according to the manufacturer's protocol for TMT kit/iTRAQ kit (Thermo Fisher). Next, TMT-labeled tryptic peptides were fractionated by high $\mathrm{pH}$ reverse-phase HPLC using a Thermo Betasil C18 column, then peptide fractions were subjected to NSI source followed by tandem mass spectrometry (MS/MS) in Q ExactiveTM Plus (Thermo) coupled online to the UPLC for identification of peptides (for proteomics) or phosphorylation sites (for phosphomics). The resulting MS/MS data were processed using Maxquant search engine (v.1.5.2.8).

\section{Assay of Mitochondrial Transmembrane Potential}

Oocytes were incubated at $37^{\circ} \mathrm{C}$ for $20 \mathrm{~min}$ with the fluorescent potentiometric indicator JC-1 (Cat\#: 40706ES60, Yeasen, Shanghai, China) diluted 1:200, then washed twice with PBS and added to droplets $(50 \mu \mathrm{l})$ of culture medium. Images of green fluorescence (JC-1 as monomers at low membrane potentials) and red fluorescence (JC-1 as aggregates at higher membrane potentials) were captured using confocal microscopy as above. Mitochondrial depolarization is indicated by a 
decrease in the red/green fluorescence intensity ratio.

\section{Detection of ROS Generation}

The ROS Assay Kit (Cat\#: S0033, Beyotime, Beijing, China) was used to detect ROS generation in oocytes. In brief, oocytes were incubated with a dichlorofluorescein diacetate (DCFH-DA) probe for $20 \mathrm{~min}$ at $37^{\circ} \mathrm{C}$ in the dark, washed, and mounted on slides for confocal imaging.

\section{RNA Sequencing and Analysis}

RNA samples were collected from mouse ovaries. RNA isolation, library construction, and RNA sequencing (RNA-seq) were carried out by the Beijing Genomics Institute following standard protocols. The library products were sequenced using a BGISEQ-500. Standard bioinformatics analysis was performed by the Beijing Genomics Institute. For gene expression analysis, the significance of the differential expression genes was defined by the bioinformatics service of BGI according to the combination of the absolute value of $\mid \log 2$ (treated/control) $\mid \geq 2$ and $q$ value $<0.001$. All original sequence datasets have been submitted to the database of NCBI Sequence Read Archive (SRA) under accession number.

\section{RNA-Protein Crosslinking and Gel Shift Assay}

To determine whether p-Bin2 or p-RPS6 binds the 5'UTR region, and to meticulously analyze which sub-region of the 5'UTR is preferentially bound, we used two different scales of subdivision (Figure 5M). For one scale, we sub-divided the whole 5' UTR of NNT into seven sub-regions (each are 20-30 bps long); for another scale, we sub-divided the whole 5' UTR of NNT into four sub-regions (each are 40-50 bps long).

Short single-strand RNAs were produced by using the T7 Ribomax Express RNAi System (Promega, Madison, WI, USA) according to the manufacturer's instructions. In brief, for each single-strand RNA, a pair of synthesized complementary single-stranded DNA oligonucleotides were first annealed to form a double-stranded DNA template (Supple. Table 3). Subsequently, the single-stranded RNA was 
synthesized in accordance with the templates. RNA was purified by conventional phenol/chloroform/isopropanol precipitation, then aliquoted and stored at $-80^{\circ} \mathrm{C}$ after a quality check on an agarose gel.

p-Bin2 or p-RPS6 were incubated for 20 minutes with each of the seven shorter sub-regions or each of the four longer sub-regions. After incubation, the mixtures were placed on a parafilm-coated plate on top of an ice plate, then UV-irradiated three times using CL-1000 UV Crosslinker (Upland, CA, USA) at $254 \mathrm{~nm}, 5 \mathrm{~mJ} / \mathrm{cm}^{2}$ energy. Next, each crosslinked complex was separated on a $0.6 \%$ agarose gel side-by-side. The corresponding RNA alone was also loaded as a control.

\section{Statistical Analysis}

All experiments were repeated at least three times. Data are presented as mean \pm SEM. Comparisons between two groups were made by Student's $t$ test. Differences among more than two groups were compared using one-way ANOVA. $\mathrm{P}<0.05$ was considered statistically significant. Statistical analyses were conducted with GraphPad Prism. 

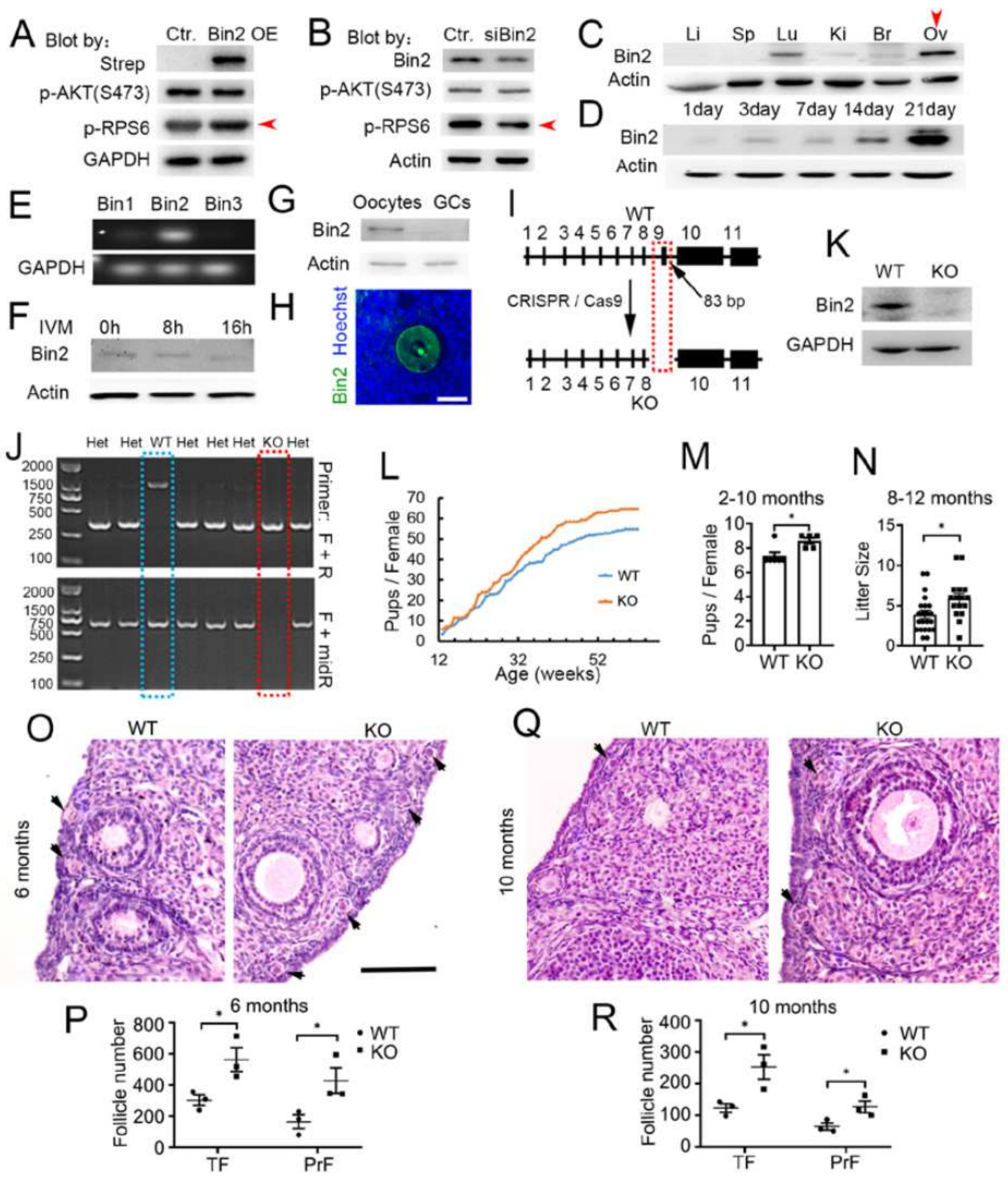

\section{Figure 1}

A. Western blot showed that Bin2 overexpression in human epithelial ovarian cancer (EOC) cells A2780 significantly up-regulated p-RPS6 (red arrow) of mTOR pathway, while not altering p-Akt. B. Western blot showed that Bin2 knockdown in A2780 significantly down-regulated p-RPS6 (red arrow) of mTOR 
pathway, while not altering p-Akt. C. Western blot showed that Bin2 protein was the richest in ovarian tissue (arrow pointed), compared with other major tissues. Li, liver; $\mathrm{Br}$, brain; Ov, ovary; Sp, spleen; Ki, kidney; Lu, lung. D. Bin2 protein level sharply increased as the initial follicle recruitment happened (around PND 21). PND, post-natal-day. E. RT-PCR in oocytes showed that the Bin2 mRNA level was much higher than the levels of another two Bin family members, Bin1 and Bin3. F. Western blot showed that Bin2 level remained constant during meiosis. $\mathrm{G}$ and $\mathrm{H}$. Western blot and immunofluorescence showed that Bin2 protein was more dominant in oocytes than in granulosa cells. I and $\mathrm{J}$. We used cas 9 technique to delete the entire exon 9 ( 83 bases, red rectangle-circled) of Bin2 genome to obtain Bin2 global knockout (KO) mice, western blot showed that Bin2 protein completely disappeared in the Bin2-knockout ovaries. K. Genotypying of Bin2-KO mice through PCR and DNA gel electrophoresis. Blue rectangle indicates WT mouse, red rectangle indicates Bin2-KO mouse. L-N. 60-week-long fertility assay showed that in Bin2-KO group, the cumulative litter number per mouse significantly increased $(L)$; the average litter numbers per female from 2 to 10 months significantly increased (M); and the average litter size (pups/litter) from 8 to 12 months also significantly increased (N). O-R. Continuous follicle count showed that at 6 months old $(\mathrm{O}$ and $\mathrm{P})$ or 10 months old $(\mathrm{Q}$ and $\mathrm{R})$, total follicle numbers and primordial follicle numbers significantly increased in Bin2-KO ovaries. Scale bar for $\mathrm{H}, 50 \mu \mathrm{m}$; scale bar for $\mathrm{O}$ and $\mathrm{Q}, 100 \mu \mathrm{m}$. * indicates $\mathrm{p} \otimes 0.05$.
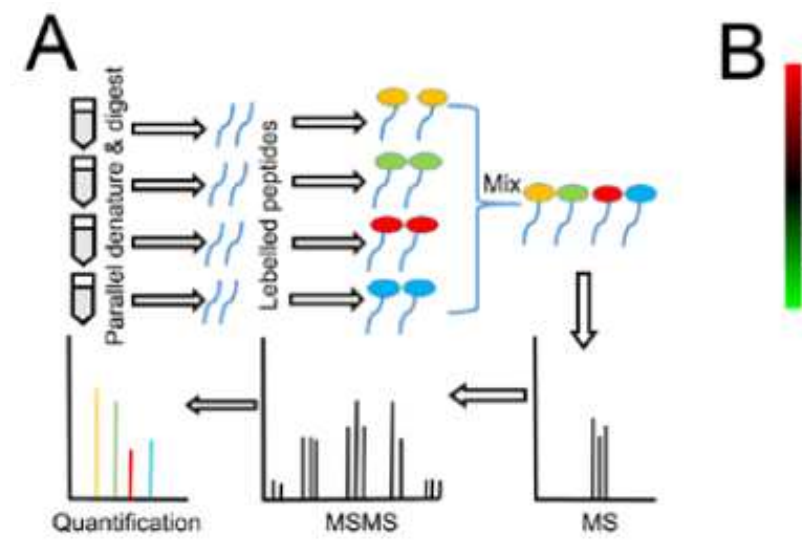
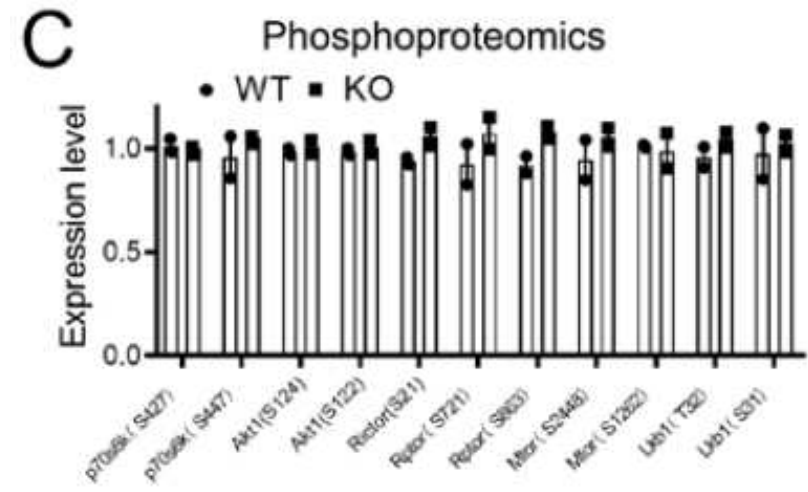

G

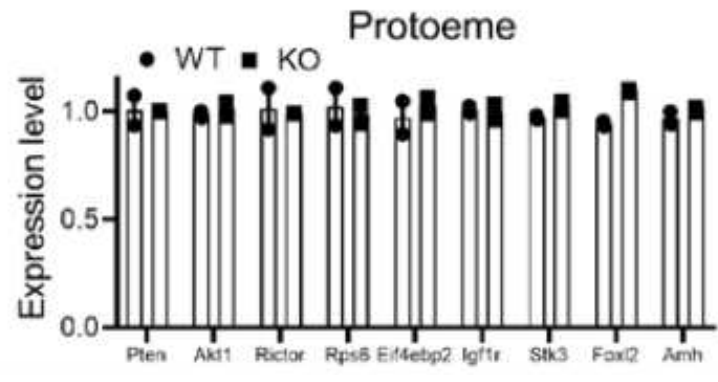

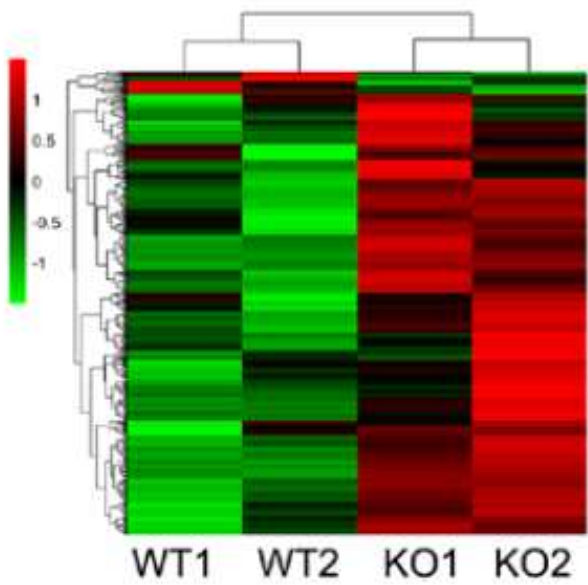

D

P-RPS6

RPS6

P-AKT

AKT

Actin
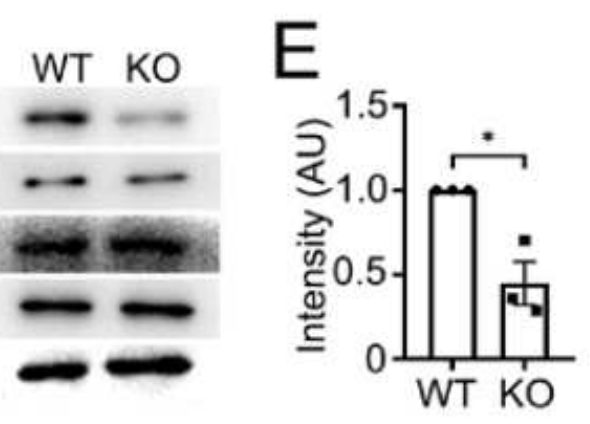

F

P-RPS6

Hoechst

WT

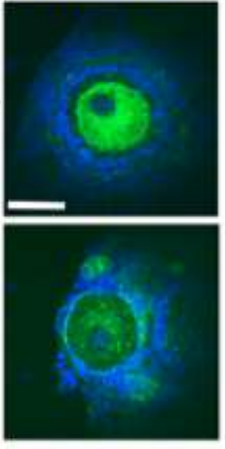

RNAseq

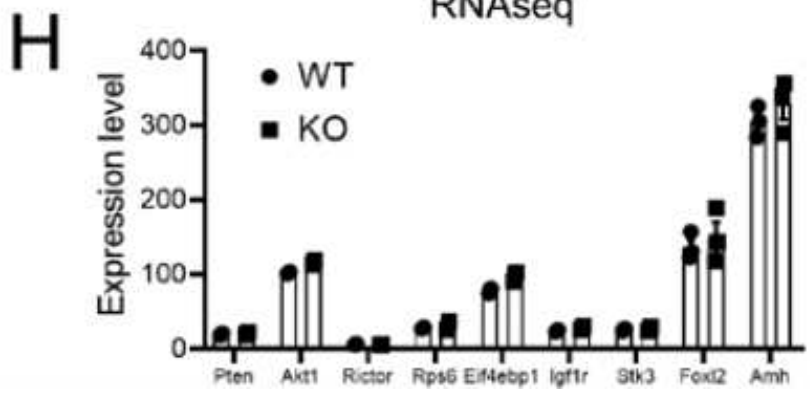




\section{Figure 2}

A. We used TMT-labeled quantitative phosphomics to systematically identify differentially phosphorylated proteins ( $\geq 1.2$ fold or $\leq 0.833$ fold) between control and Bin2-KO 3-week-old ovaries. B and $\mathrm{C}$. We found that among mTOR pathway proteins, only RPS6 had significantly decreased phosphorylation. D-F Both western blot and immunofluorescence showed that among mTOR pathway proteins, only RPS6 had significantly decreased phosphorylation in Bin2-KO ovaries. G. TMT-labeled quantitative gross proteomics showed that the gross protein level of each mTOR pathway component was unaltered. H. RNA sequencing showed that the mRNA level of each mTOR pathway component (pTEN, Akt1, Rictor, Rps6, Eif4ebp1, IGF1R, Stk3, Foxl2, and Amh) was unaltered. Scale bar, $50 \mu m$. * indicates $p \otimes 0.05$.

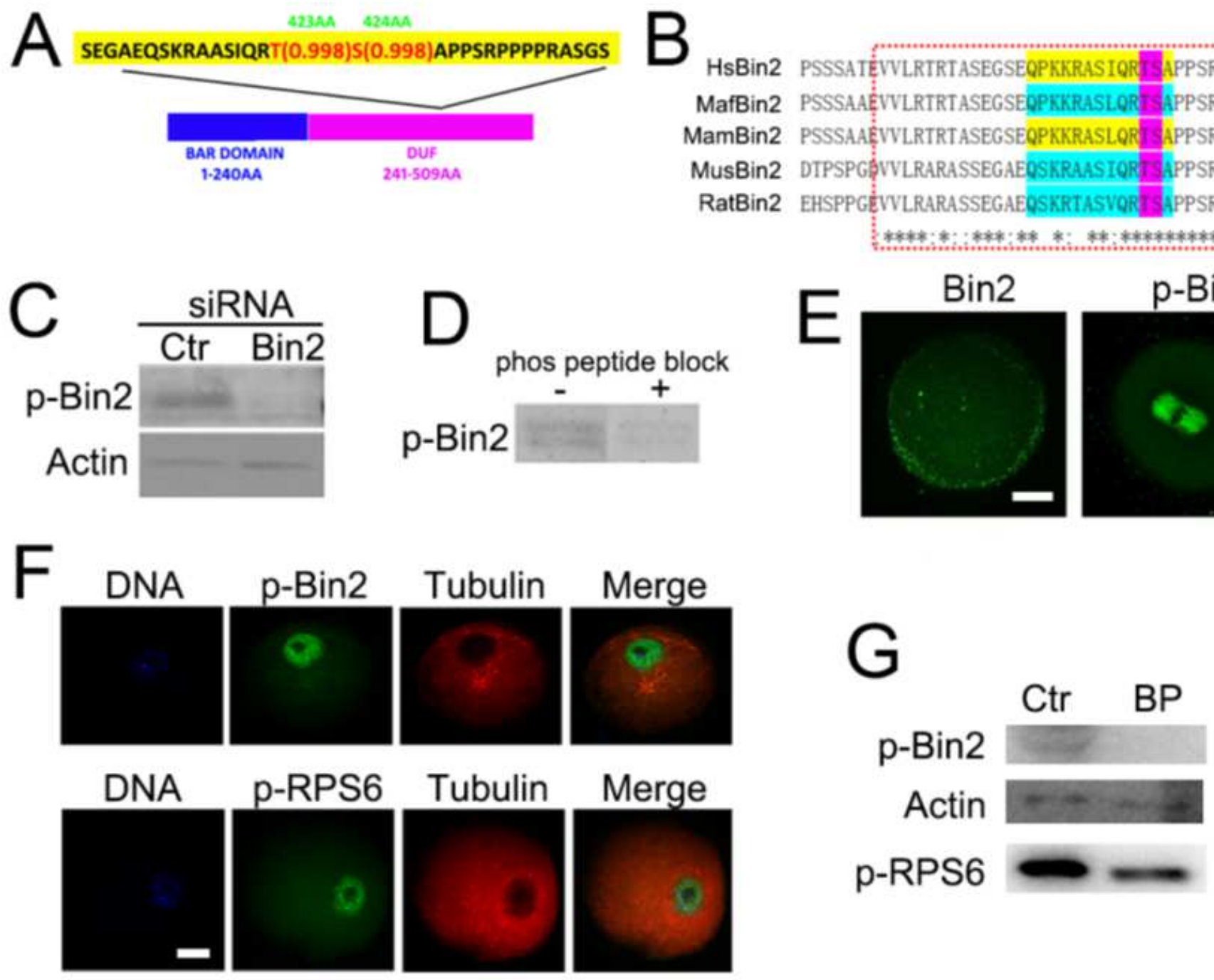

\section{Figure 3}

A. We identified several phosphorylation sites (Supplementary table 1); among these, Thr423 \& Ser424 were in the c-terminal domain of unknown function (DUF domain). B. Thr423 \& Ser424 and the adjacent residues are highly conserved between humans, rats, mice, rhesus monkeys, and cynomolgus macaques. C and D. We hypothesized that Thr423 \& Ser424 are the key residues for the activation and translocation 
of Bin2. We generated a phosphorylation antibody using a core phosph peptide "QSKRAASIQR(pThr) (pSer)A" and verified its specificity through Bin2 siRNA and corresponding non-phosph peptide "QSKRAASIQRTSA." E. Immunofluorescence showed that Bin2 was enriched on oocyte membrane, while p-Bin2 was enriched within spindles during meiosis. F. Immunofluorescence showed that p-Bin2 was enriched within the nucleus of GV oocytes, similar to p-RPS6. G. We injected the non-phosph peptide "QSKRAASIQRTSA" into GV oocytes and found that the p-Bin2 level significantly decreased, while the pRPS6 level also significantly decreased. Scale bar, $20 \mu \mathrm{m}$.
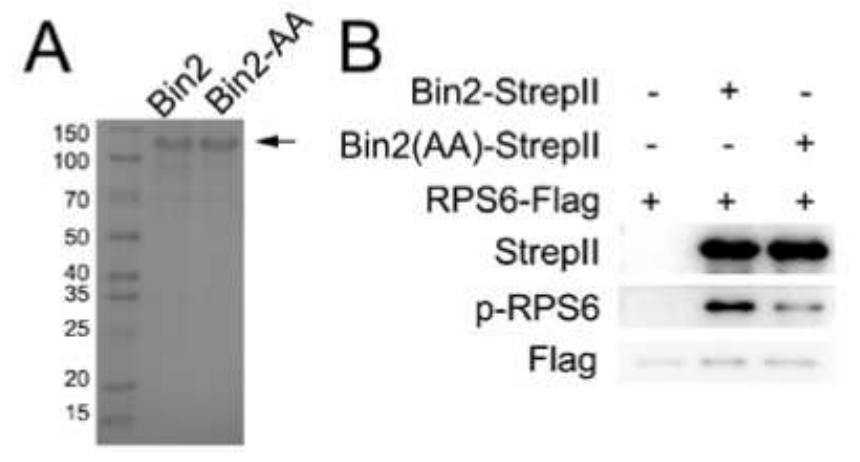

Flag
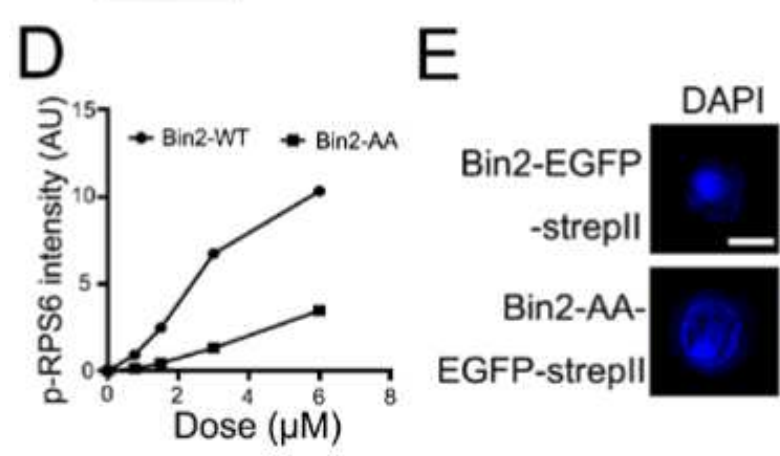

$\mathrm{H}$

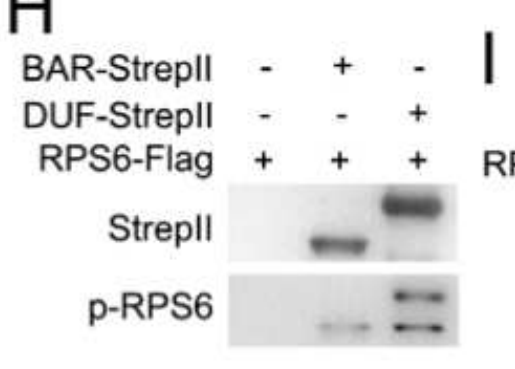

EGFP-strepII

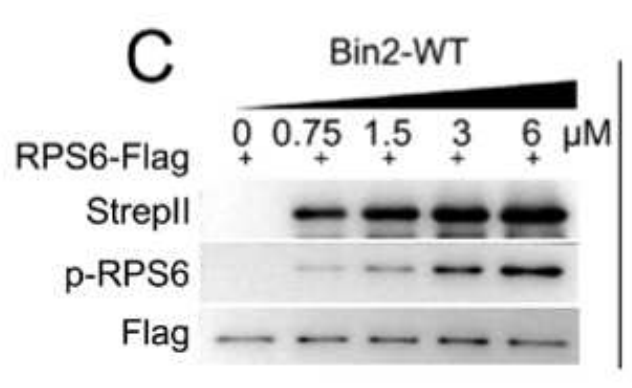

Flag
Bin2-AA

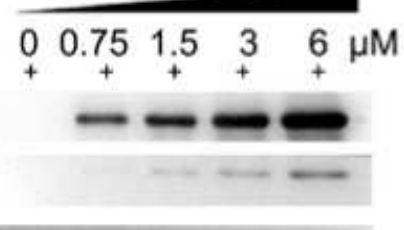

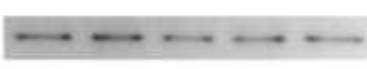
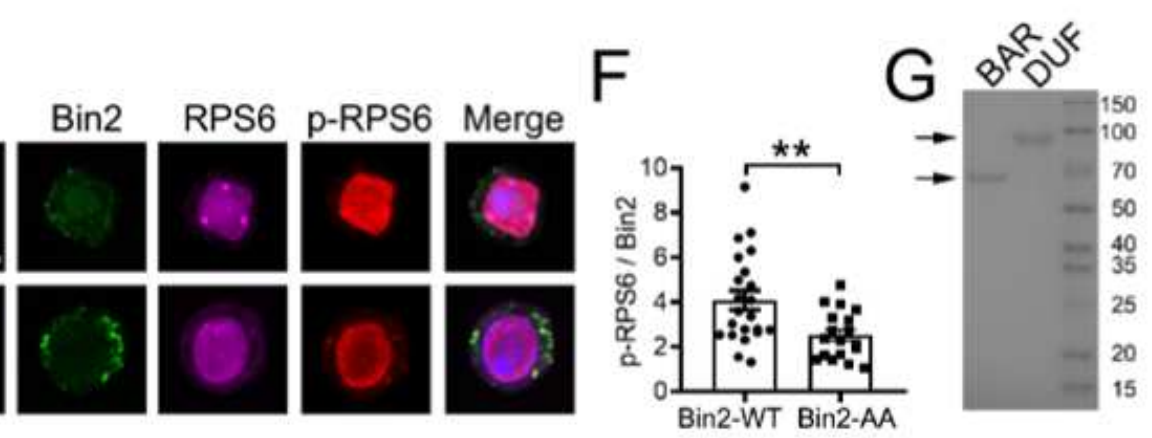

\section{Figure 4}

A. We used the Bac-to-bac system to express full-length wild-type Bin2 (Bin2-WT) and a Bin2-AA mutant (Thr423 \& Ser424 to Ala423 \& Ala 424). SDS-PAGE and commassie staining showed that both purified proteins had good purity and expected size. B. In-vitro phosphorylation and western blot showed that Bin2-WT can phosphorylate RPS6 more than an equal amount of Bin2-AA. C. In-vitro, dose-dependent phosphorylation assay and western blot showed that Bin2-WT could phosphorylate RPS6 much more strongly than Bin2-AA. D. Quantification of C. E. Immunostaining on bacularvirus-infected sf9 cells showed that Bin2-WT localized both on the membrane and within the cytoplasm, while Bin2-AA almost exclusively localized on the membrane. Accordingly, cytoplasmic p-RPS6 intensity in Bin2-WT bacularvirus-infected infected sf9 cells was significantly higher than in Bin2-AA bacularvirus-infected sf9 
cells. F. Quantification of E. G. We expressed and purified two different motifs of Bin2, BAR (1-240 AA) and DUF (domain of unknown function, 241-505 AA); SDS-PAGE and commassie staining showed that both purified proteins had good purity and expected size. H. In-vitro phosphorylation and western blot showed that Bin2-DUF can phosphorylate RPS6 more than an equal amount of Bin2-BAR. I. In-vitro dosedependent phosphorylation assay and western blot showed that Bin2-DUF could phosphorylate RPS6 much more strongly than Bin2-BAR. J. Quantification of I. Scale bar, $10 \mu \mathrm{m}$. ** indicates $\mathrm{p} \otimes 0.01$.

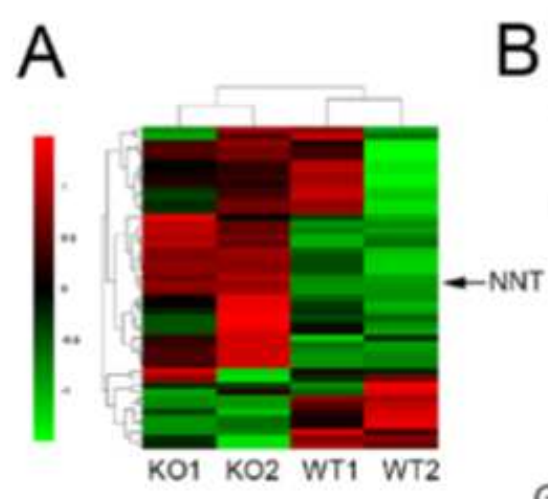

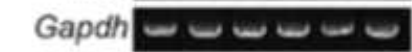
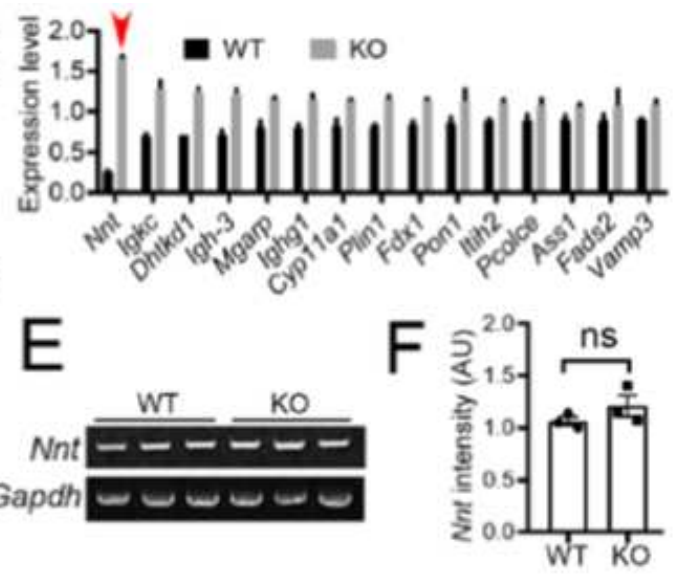
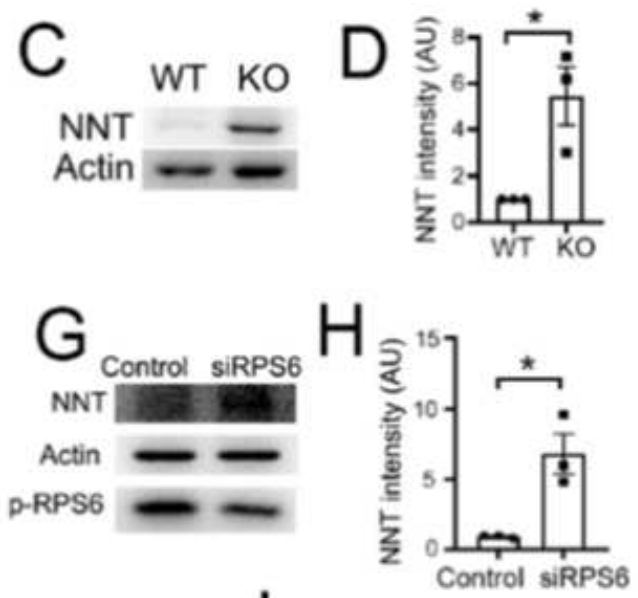
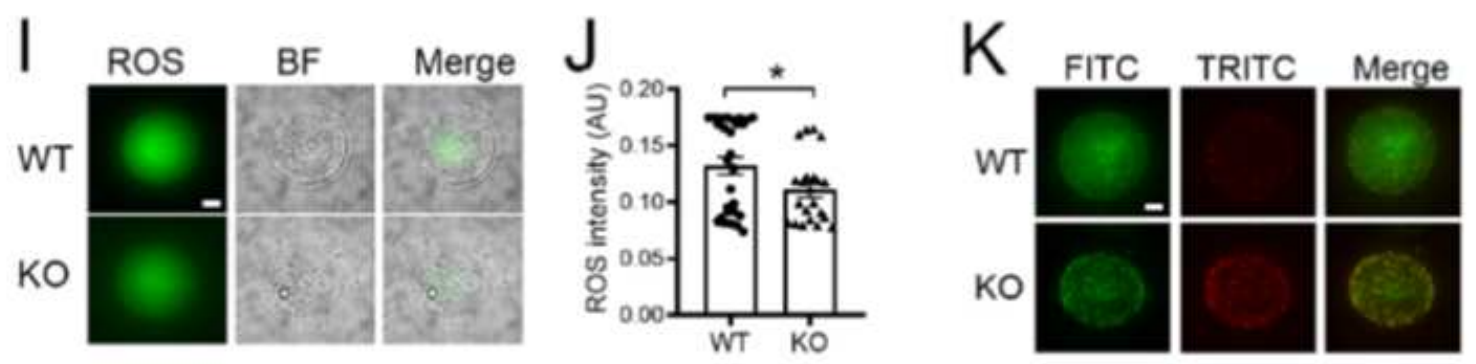

L

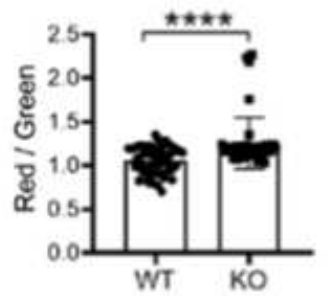

M
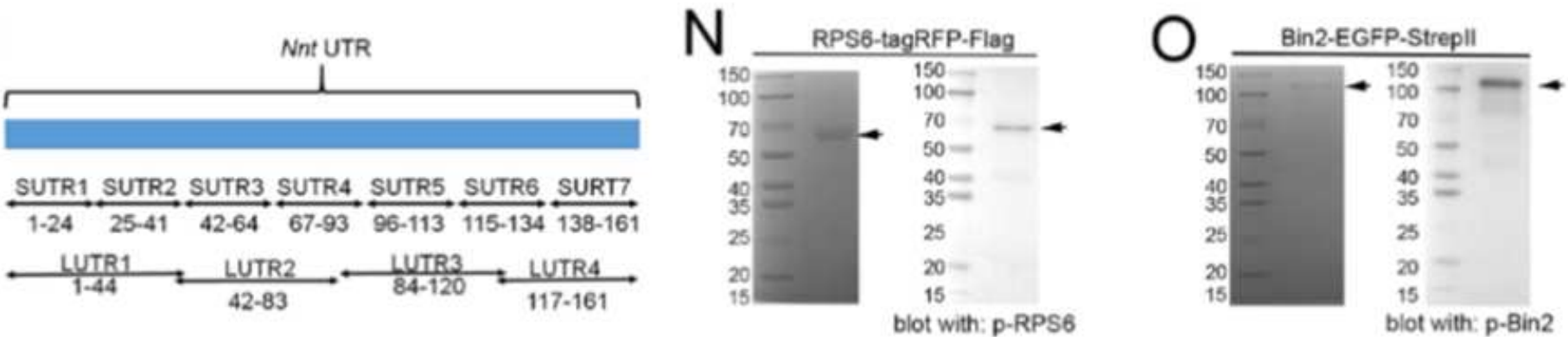

$\mathrm{P}$

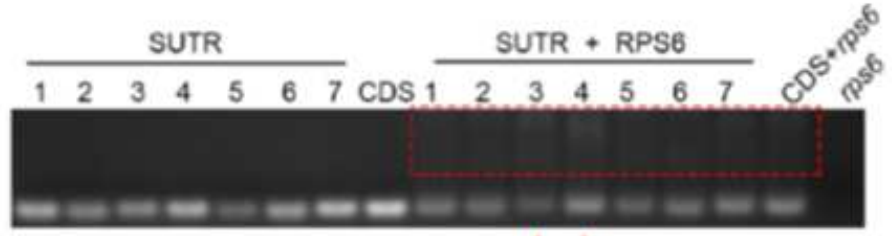

\section{$A$ A}

R SUTR
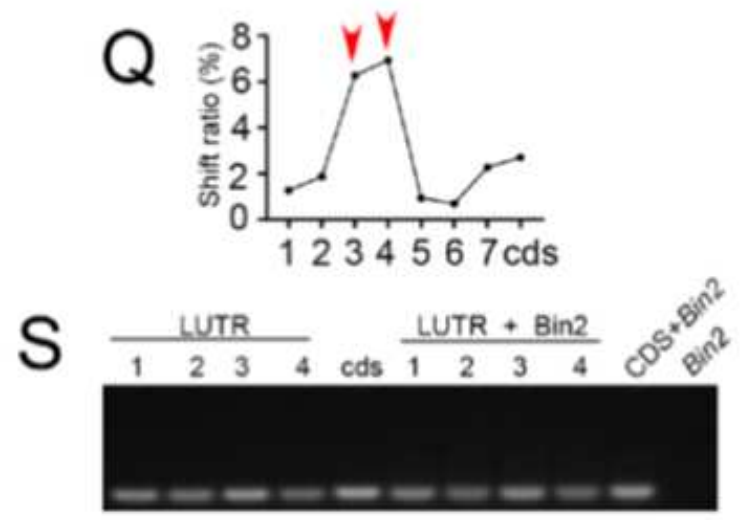
A and B. We used TMT-labeled quantitative proteomics to systematically identify differentially expressed gross proteins between control and Bin2-knockout, 3-week-old ovaries ( $\geq 1.2$ fold or $\leq 0.833$ fold). Among the differentially expressed gross proteins, nicotinamide nucleotide transhydrogenase (NNT) was most up-regulated ( $>6$ fold, red-arrow pointed). $C$ and $D$. Western blot and quantification verified that the NNT protein level in the Bin2-knockout group increased more than 6-fold compared to control. E and F. RT-PCR and quantification showed that Bin2 knockout did not affect the mRNA level of NNT. G and H. Western blot and quantification showed that the NNT protein level in the RPS6-knockdown group significantly increased. I and J. Live dye staining and quantification showed that the ROS level significantly decreased in Bin2-knockout oocytes. K and L. JC-1 staining and quantification showed that the mitochondria membrane potential significantly increased in Bin2-knockout oocytes. M. We attempted to address whether p-Bin2 or p-RPS6 bind the translation-regulating regions of NNT to inhibit its translation. We subdivided NNT 5' UTR into seven shorter or four longer regions and in-vitro transcribed single-strand RNA of these regions. $\mathrm{N}$ and $\mathrm{O}$. SDS-PAGE, commassie staining, and western blot showed that RPS6 (N) and Bin2 (0) proteins had phosphorylated portions. P and Q. Protein-RNA UV crosslinking and gel shift assay showed that p-RPS6 prefer to bind short region three and four, from 42 to $95 \mathrm{bp}$ of NNT 5' UTR (redarrow pointed), instead of other UTR regions or CDS region. Q. Quantification of P. R and S. Protein-RNA UV crosslinking and gel shift assay showed that $\mathrm{p}$-Bin2 did not bind any shorter or longer regions. Scale

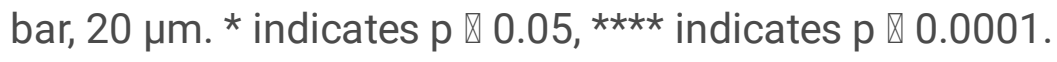



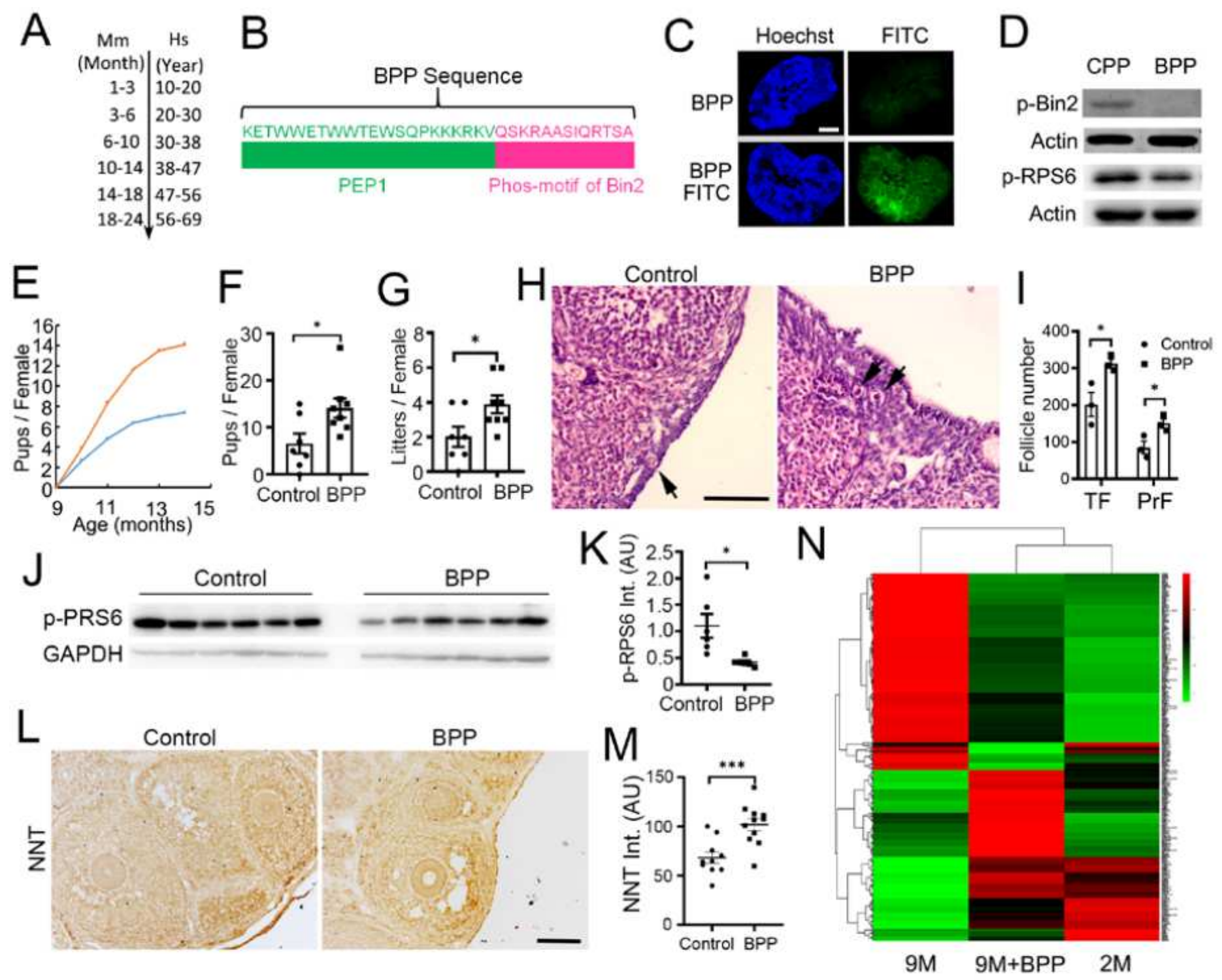

0
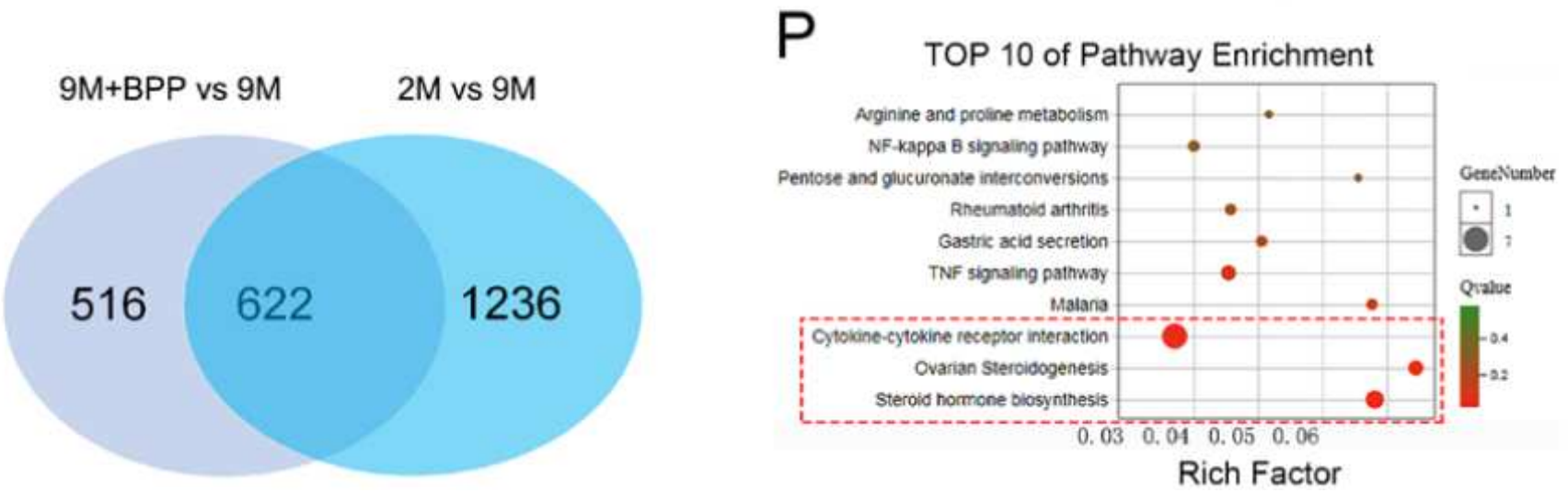

\section{Figure 6}

A. Analogical time axis between mouse and human. We planned to use 8-month-old aging mice as models to mimic 35-year-old women. B. To facilitate the permeation of this peptide in vivo, we fused PEP1, the known cell-penetrating peptide, to the non-phosphor Bin2 peptide "QSKRAASIQRTSA" and renamed it as BPP (Bin2-penetrating peptide). $C$ and D. We firstly verified that FITC-labeled BPP can efficiently enter ovaries three days after injection (C), and both p-Bin2 and p-RPS6 significantly decreased 
within ovaries (D). E-G. After a 3-week-long BPP injection cycle (once a day) in 8-month-old aging mice, fertility assay from 36 weeks old to 63 weeks old showed that both the average number of cumulative pups per female ( $E$ and $F$ ) and average litters per female $(E$ and $G$ ) significantly increased. $H$ and $I$. Continuous follicle counting showed that both total follicles and PMFs significantly increased in the BPPtreated group. $\mathrm{J}$ and $\mathrm{K}$. Western blot and quantification showed that p-RPS6 significantly decreased in the BPP-treated group. L and M. Immunostaining and quantification showed that the NNT level within the oocytes significantly increased in the BPP-treated group. $\mathrm{N}$ and $\mathrm{O}$. We examined how well BPP could recover ovarian function in the aging mice at the transcriptional level. RNA sequencing showed that at the fold threshold of $\mid \log 2(2-$ month/9-month)| $\geq 2$, there were 1858 DEGs (differentially-expressed genes) between 2-month ovaries and 9-month ovaries; at the fold threshold of $\mid \log 2$ (9-month-BPP/9-month) $\mid \geq 2$, there were 1138 DEGs (differentially-expressed genes) between 9-month-BPP ovaries and 9-month ovaries; and between these two clusters of DEGs, 622 DEGs overlapped. P. KEGG analysis of functionallyannotated DEGs showed that multiple follicle survival, oocyte quality, or oocyte steriodgenesis-related pathways are among the top 10 enriched signaling pathways. Scale bar for B, $200 \mu \mathrm{m}$; scale bar for $\mathrm{H}$ and $\mathrm{L}, 100 \mu \mathrm{m}$. * indicates $\mathrm{p} \otimes 0.05$.
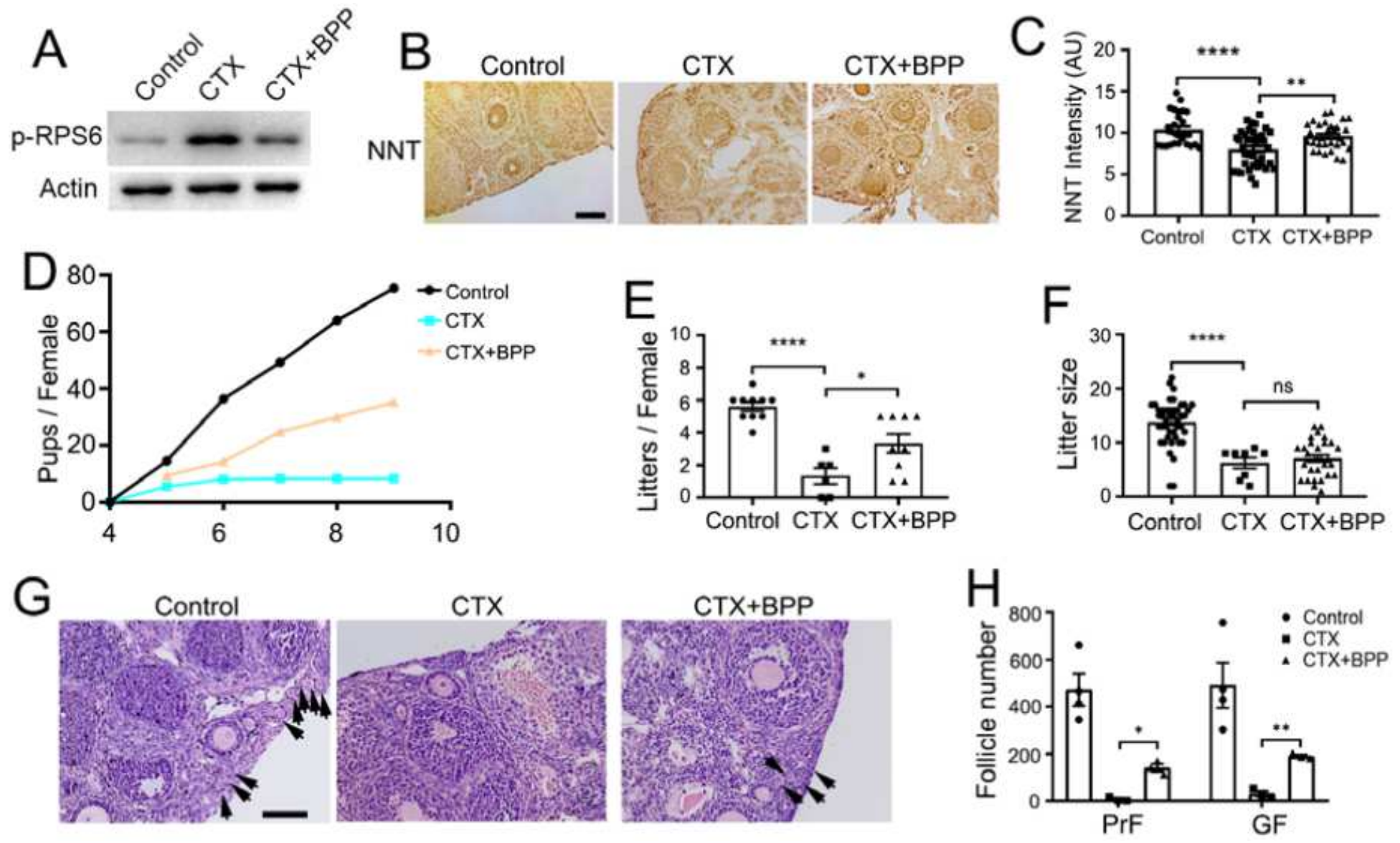

Figure 7

A. Western blot showed that a harsh, three-week-long cyclophosphamide (CTX) daily treatment significantly elevated p-RPS6, while BPP treatment reduced the p-RPS6 level close to control level. B and C. Immunostaining and quantification showed that CTX treatment significantly decreased the oocyte 
NNT level, while BPP injection recovered the NNT level close to control. D-F. Fertility assay from 4 months old to 9 months old showed that CTX treatment caused almost completed infertility, while BPP significantly recovered fertility (although still less than control). $\mathrm{G}$ and $\mathrm{H}$. Continuous follicle counting showed that CTX treatment sharply decreased follicle numbers at each stage, while BPP could significantly increased follicle numbers at each stage (although still less than control). Scale bar, $100 \mu \mathrm{m}$. * indicates $p \otimes 0.05$, ** indicates $p \otimes 0.01, * \star * *$ indicates $p \otimes 0.0001$.

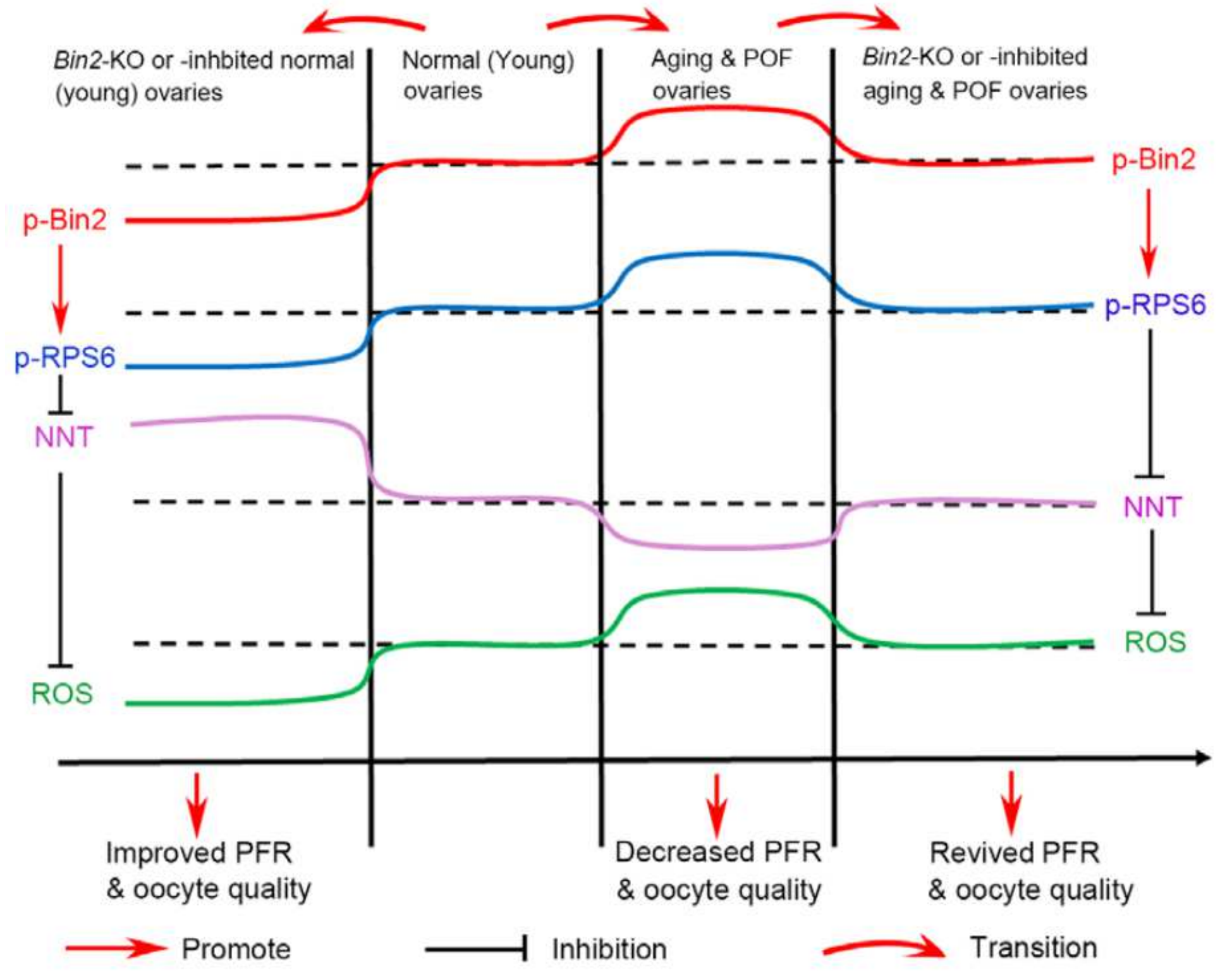

Figure 8

In this model, the correlations of primordial follicle and oocyte quality with the level of p-Bin2, p-RPS6, NNT, and ROS are shown under four conditions. Two adjacent conditions are isolated by a vertical axis representing the suppositional protein levels. From left to right, Bin2-KO or -inhibited normal (young) ovaries, normal (young) ovaries, aging \& POF ovaries, and Bin2-KO or -inhibited aging \& POF ovaries. The red curved arrows on the top indicated the transitions from one condition to the other. Four solid colored horizontal lines (red for p-Bin2, blue for p-RPS6, magenta for NNT, and black for ROS ) connected by 
dotted line indicate the suppositional normal level of these four substances. Upon transition from normal (young) ovaries to Bin2-KO or -inhibited normal (young) ovaries, p-Bin2 is downregulated and conduced to p-RPS6 downregulation, NNT upregulation, and ROS downregulation, which eventually improve PFR and oocyte quality. Upon transition from normal (young) ovaries to aging \& POF ovaries, p-Bin2 is upregulated and conduced to p-RPS6 upregulation, NNT downregulation, and ROS upregulation, which eventually decrease PFR and oocyte quality. Upon transition from aging \& POF ovaries to Bin2-KO or inhibited aging \& POF ovaries, higher p-Bin2 is downregulated close to normal level and conduced to higher p-RPS6 downregulated close to normal level, lower NNT upregulated close to normal level, and higher ROS downregulated close to normal level, which eventually revive PFR and oocyte quality.

\section{Supplementary Files}

This is a list of supplementary files associated with this preprint. Click to download.

- ZhuFYetalSupplementaryFigureswithLegends.pdf 